\author{
UNITED STATES \\ DEPARTMENT OF THE INTERIOR \\ GEOLOGICAL SURVEY \\ WASHINGTON 25, D.C.
}

AUG 121953

AEC $-50 / 4$

Dr. Phillip I. Merritt, Assistant Director

Division of Raw Materials

U. S. Atomic Energy Commission

P. 0. Box 30, Ansonia station

New York 23, New York

Dear Phil:

Transmitted herewth are six copies of TEI-334, "Identification

and occurrence of uranium and vanadium minerals from the Colorado Plateaus," by A. D. Weeks and M. E. Thompson, Apri1 1953.

We are asking Mr. Hosted to approve our plan to publish this

report as a Circular.

Sincerely yours,

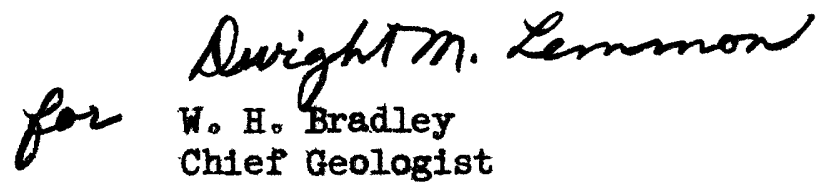


UNITED STATES DEPARTMENT OF THE INTERIOR

GEOLOGICAL SURVEY

Geology and Mineralogy

This document consists of 69 pages. Series A.

IDENTIFICATION AND OCCURRENCE OF URANIUM AND VANADIUM MINERAIS

FROM THE COLORADO PIATEAUS*

By

A. D. Weeks and M. E. Thompson

April 1953

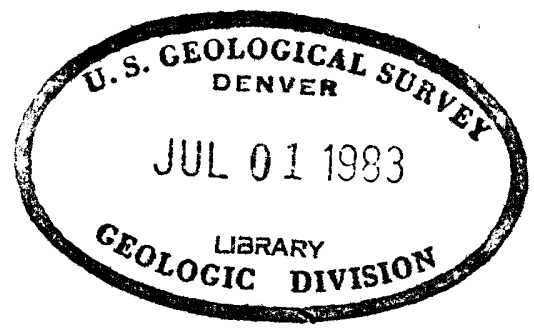

Trace Elements Investigations Report 334

This preliminary report is distributed without editorial and technical review for conformity with official standards and nomenclature. It is not for public inspection or quotation. 
USGS - TEI -334

GEOLOGY AND MINERALOGY

Distribution (Series A)

No. of copies

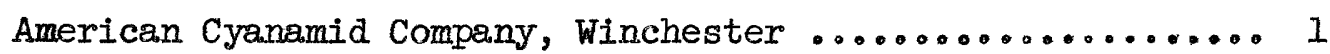

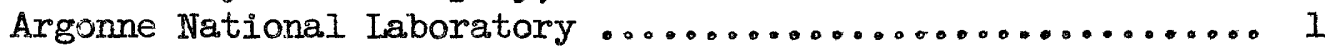

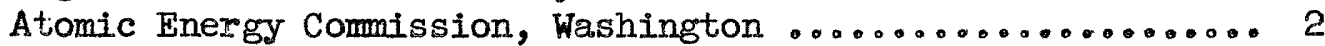

Battelle Memorial Institute, Columbus .............. I

Carbide and Carbon Chemicals Company, Y-12 Area ........ I

Division of Raw Materials, Butte .................... 1

Division of Raw Materials, Denver ................... 1

Division of Raw Materials, Grants .................. I

Division of Raw Materials, Hot Springs ................ 1

Division of Raw Materials, New York .................. 6

Division of Raw Materials, Richfield .................. I

Division of Raw Materials, Salt Lake City ............. I

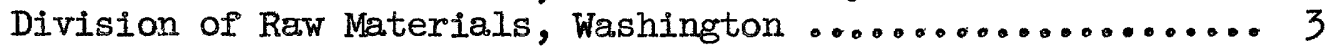

Division of Research, Washington .................... I

Dow Chemical Company, Pittsburg .................... 1

Exploration Division, Grand Junction Operations Office ..... 6

Grand Junction Operations Office ..................... 2

Technica.1 Information Service, Oak Ridge .............. 6

Tennessee Valley Authority, Wilson Dam ................ 1

U. S. Geological Survey:

Alaskan Geology Branch, Washington .................. 1

Fuels Branch, Washington ......................... 6

Geochemistry and Petrology Branch, Washington ............. 30

Geophysics Branch, Washington ...................... I

Mineral Deposits Branch, Washington .................. 3

E. H. Bailey, San Francisco ......................... I

J. R. Cooper, Denver ............................ I

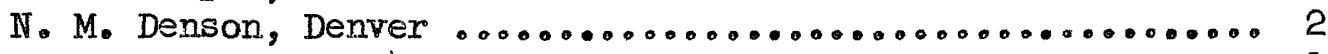

C. E. Dutton, Madison ............................ 1

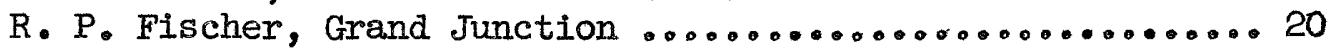

I. S. Gardner, Albuquerque ....................... 3

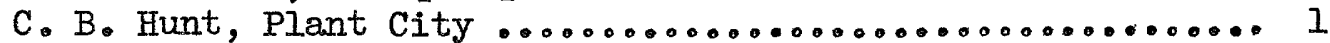

M. R. Klepper, Washington $\ldots \ldots \ldots \ldots \ldots \ldots \ldots \ldots \ldots \ldots \ldots \ldots \ldots \ldots$ I

A. H. Koschmann, Denver .......................... I

R. A. Laurence, Knoxville ....................... I

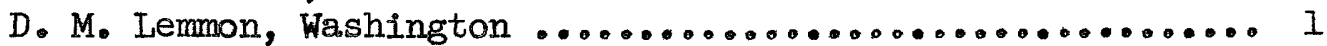

J. D. Love, Laramie ............................. 1

L. R. Page, Denver ............................... 20

R. J. Roberts, Salt Lake City ....................... I

Q. D. Singewald, Beltsville ..................... 1

J. F. Smith, Jr •, Denver ......................... 1

R. W. Swanson, Spokane .......................... I

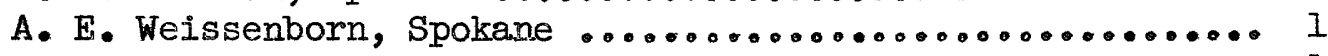

W. P. Williams, Joplin .......................... I

TEPCO, Washington:

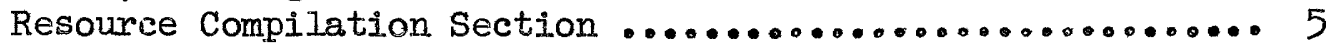

Reporta Processing Section ........................ 5

(Including master) 
CONIENTS

Page

Part I

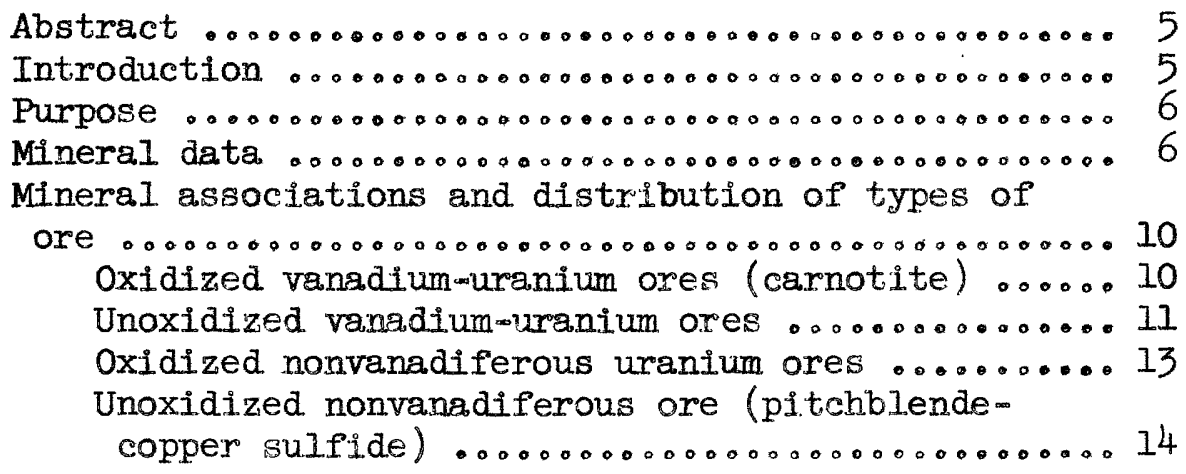

Part II

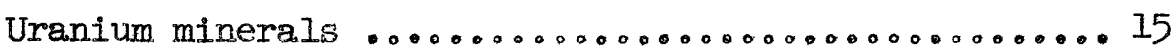

Description of identified minerals ........... 15

Autunite and meta-autunite $I \ldots \ldots \ldots \ldots \ldots \ldots 17$

Bassetite ....00.0000000000000.000.0...18

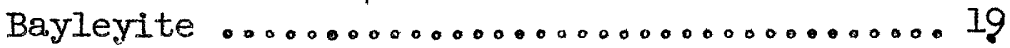

Becquerelite $0000000000000000000 \ldots 00 \ldots 20$

Carnotite o00000000000000000000000000002I

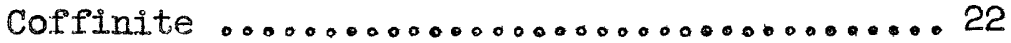

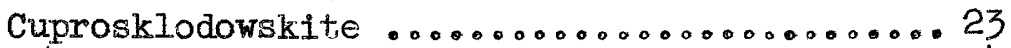

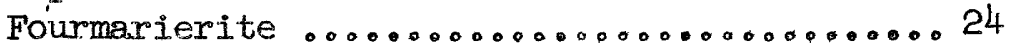

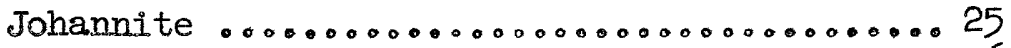

I.iebigite $0000000000000000000000000 \ldots 26$

Novacekite 0000000000000000000000000000000027

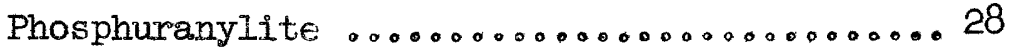

Rabbittite 0.00000000000000000000000000.29

Rauvite o00000000000000000000000000000.3 30

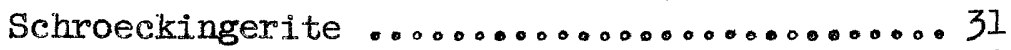

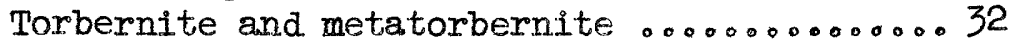

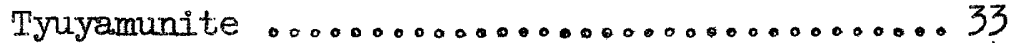

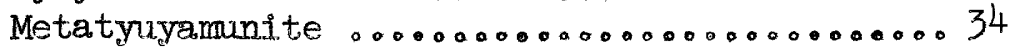

Uraninite (pitchblende) o000000000.....35

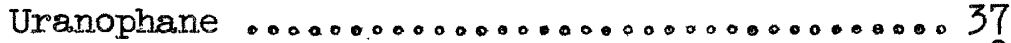

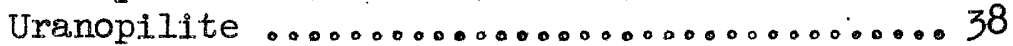

Uvanite ..000000000000000000000000000.0.039

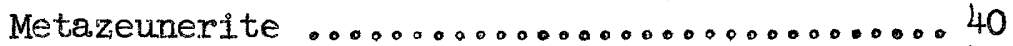

Betazippeite .0000.00000000000000000.41

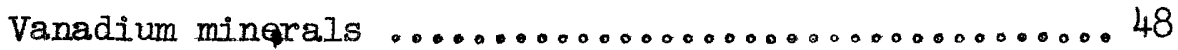

Description of identified minerals (uranyl

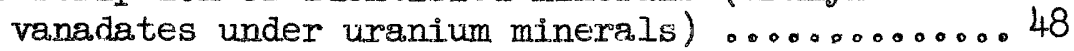

Corvusite .0.0.0.0.0.0.0.000000000.0.50

Doloresite and lumsdenite .........

Fervanite 00000000000000000000000000000000052

Hewettite .000000000000000000000000000000053 
Part II Vanadium minerals (Continued)

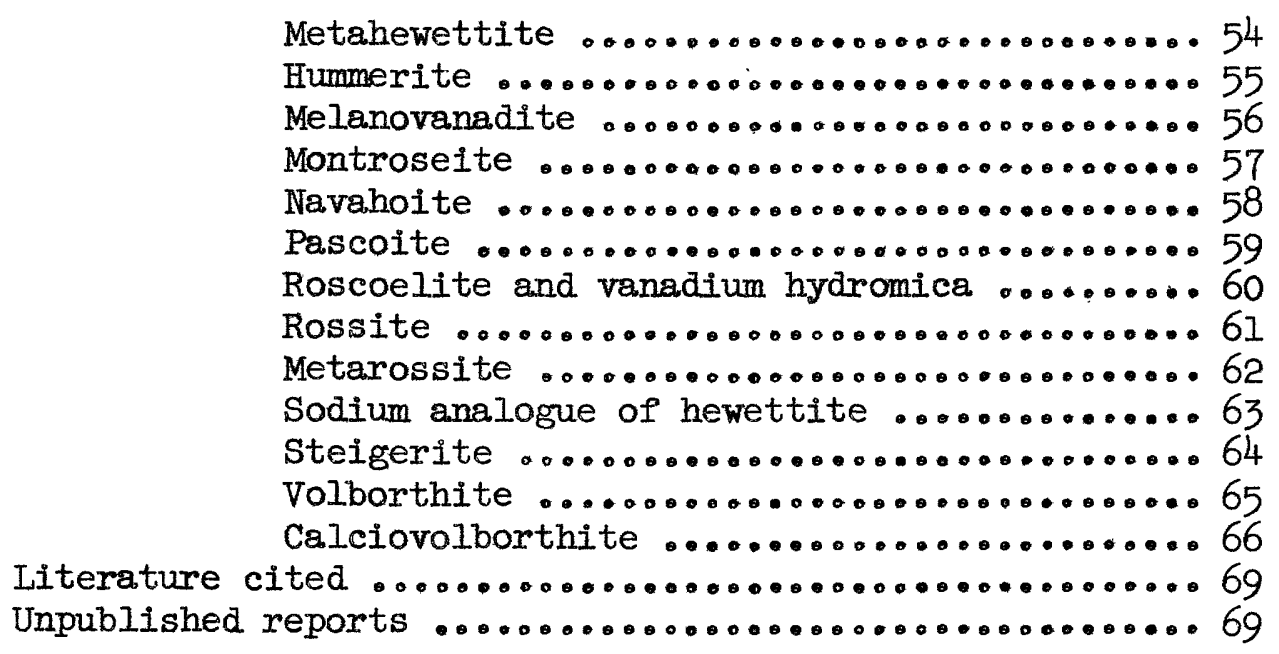

TABIES

Page

Table 1. Optical properties of uranium minerals ...... 42-48

2. List of mine names showing county and state .. 67-68 


\section{IDENTIFICATION AND OCCURRENCE OF URANIUM AND VANADIUM MINERAIS \\ FROM THE COLORADO PLATEAUS}

by A. D. Weeks and M. E. Thompson

Part I

ABSTRACT

This report, designed to make available to field geologists and others information on identification and occurrence of uranium minerals of the Colorádo Plateaus, contains physical properties, $X$-ray data, and in some instances results of chemical and spectrographic analysis of 24 uranium and 17 vanadium minerals. Also included is a table giving the optical properties of uranium minerals and a list of locations of mines from which the minerals have been identified.

\section{INTRODUCTION}

More than 20 uranium and about 20 vanadium minerals have been identified during recent mineralogic studies of uranium ores from the Colorado Plateaus. This work is part of a program undertaken by the Geological Survey on behalf of the Atomic Energy Commission.

Thanks are due many members of the Geological Survey who have worked on one or more phases of the study-including chemical, spectrographic, and X-ray examination as well as collecting of samples. We are grateful to George Switzer of the U. S. National Museum and to Clifford Frondel of Harvard. University who kindly loaned type mineral specimens and discussed various problems. 
PURPOSE

The purpose of this report is to make available to field geologists and others, who do not have extensive laboratory facilities, present information on the identification and occurrence of the uranium and vanadium minerals of ores from the Plateaus. Distinctive properties of each mineral are listed to encourage and facilitate identification by optical or chemical tests. A combination of data from $X$-roy powder patterns and spectrographic analyses is useful and efficient for cextain minerals, especially if the quantity of mineral is very small, but for many minera.ls these techniques are not necessary.

\section{MINERAL DATA}

The minerals include several new species and many that were rare and incompletely (or inaccurately) described before the recent intensive search for uranium. The data for each mineral include the best available formula, .in general from the Glossary of uranium - and thorium-bearing minerals (Frondel and Fleischer, 1952), and crystallographic and physical properties from Dana's System of mineralogy, 7 th edition, and from Mineralogy of uranium and thorium minerals (George, 1949). For minerals showing a considerable range of properties, selection has been made to emphasize those noted. by the writers for specimens from the Colorado Plateaus. Crystallographic data are reduced to a minimum because such would be used rarely by the field geologist. An exception is crystal habit which may be observed with a binocu.lar microscope, such as the platy habit of the torbernite group and bladed or fibrous habit of uranophane. Chiefly to establish the particular material that is referred to under each species, the d-spacing in Angstrom 
units is given for several strong lines of the X-ray diffraction powder pattern taken with CuKa radiation. The relative intensity of the lines is indicated by the abbreviations: VS very stong, S strong, M medium, and Weak. The X-ray photographs were taken by E. A. Cisney, and M. E. Thompson.

Relatively few of the uranium and vanadium minerals are too fine grained, too high in refractive index, or too dark for determination of some optical properties. Certain minerals, notably the torbernite group and the carnotite group, dehydrate easily with resultant rise in refractive indices; identification of minerals in these groups by optical properties must be made with considerable care. Table 1 gives the optical properties of uranium minerals.

So many yellow and greenishmyellow uranium minerals as well as a few yellow and greenish-yellow vanadium and copper minerals occur on the Plateaus that color is not a dependable means of identification unless combined with other properties. The color of fluorescence noted here is for minerals observed under ultraviolet light $(2537 \mathrm{~A})$ and may differ considerably from the color or degree of fluorescence observed at $3660 \mathrm{~A}$.

For the new and some of the doubtful species, chemical analyses were

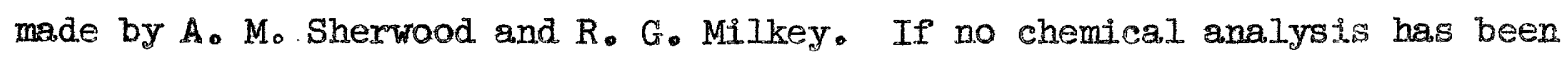
made, a spectrographic analysis is given. These are by C. L。 Waring, H. W. Worthing, C. S. Annell, J. N. Stich, and K. E. Valentine. Semiquantitative spectrographic analyses (Waring and Annell, 1952) made on $10 \mathrm{mg}$ of sample are given for constituents in the following percentage ranges: more than 10 , I to $10,0.1$ to $1,0.01$ to 0.1 , and 0.001 to 0.01 . Qualitative spectrographic analyses (Stich, 1953), ade on I mg of sample, list the constituent elements as jor (more than 10 percent), minor (approximately 1 to 10 
percent), and trace (less than 1 percent).

Under Occurrence is noted the primary or secondary nature of each mineral and whether it is found as impregnation, replacement, or coating on fractures and mine walls. Listed also are the commonly associated minerals. Only for a few rare minerals is the name of the person who collected the samples given. Most of the samples were collected by Lo $B$. Riley, I. R. Stieff, T. W. Stern, and the writers; a smaller number by other Survey geologists and by mine operators.

The section headed Identification is based on the writers' experience in identifying these minerals. Minerals that are commonly fine grained, in thin coatings, or admixed with other minerals, as are many from the Plateaus, can be identified satisfactorily in the laboratory using a small amount of material. A l-mg amount of mineral is sufficient for a spindle for an X-ray powder pattern, and the spindle may then be used for qualitative spectrographic analysis. Some groups of minerals have similar X-ray patterns, as autunite and uranocircite or metatorbernite and metazeunerite, and an additional test is necessary to determine the mineral.

A satisfactory test for uranium may be made by a bead test using a small loop of platinum wire and a flux composed of 45.5 percent by weight of $\mathrm{Na}_{2} \mathrm{CO}_{3}, 45.5$ percent by weight of $\mathrm{K}_{2} \mathrm{CO}_{3}$, and 9 percent by weight of $\mathrm{NaF}$ and observing with a long wavelength $(3650 \mathrm{~A})$ ultraviolet light the fluorescence caused by uranium (Grimaldi and others, 1952)。 The test is more easily made using a small platinum pan such as the lid of a platinum crucible. The flux should be melted and the blank tested with the ultraviolet light before the mineral grains are added and the flux remelted for the final test. With a little practice one can distinguish between the bright fluorescence of a uranium mineral and the faint fluorescence of slightly uraniferous 
material such as uraniferous opal. After obtaining a positive test the platinum wire or pan should be washed in hydrochloric acid before making another test.

The test described above may be modified by using a flux composed of nine parts of household baking soda and one part of sodium fluoride (as sold by drug stores for ant polson) and ordinary iron wire. In this case the flux should be fused only a short time to avoid adding iron that causes quenching of the uranium fluorescence. Although a wavelength of $3650 \mathrm{~A}$ is best for accurate laboratory work, almost any battery-operated. ultraviolet light suitable for prospecting may be used in this field test. To test for vanadium, dissolve a small portion of the mineral or ore in aqua regia, evaporate to dryness, add as much water as original acid, and then add a few drops of hydrogen peroxide. If vanadium is present the solution will turn orange red. Carnotite or tyuyamunite commonly turns red brown when a drop of concentrated hydrochloric acid is added but this test is not always satisfactory for roscoelite ore.

Localities are listed by mine name and the mining district, as shown on a map by Shoemaker and Luedke (1952). Most mine names are those in use when samples were collected in the summer of 1952, but some are as recorded with samples collected in 1950 and 1951. To help the reader who is not familiar with the mining districts an alphabetical list of mine and locality names is given in table 2 showing the county and state in which each is located. The number of localities is restricted to those from which specimens have been identified by the authors and to the type localities of minerals named from the Plateaus. In adation, a few samples from the sandstone-type deposits at Pumpkin Buttes, Wyo, have been included. 
MINERAL ASSOCIATIONS AND DISTRIBUTION OF TYPES OF ORE

Ore from the Plateaus may be classified on the basis of whether uranium is associated with vanadium or with copper and other metals. Each may be subdivided into highly oxidized or relatively unoxidized ore.

Oxidized vanadium-uranium ore (carnotite)

For many years the chief ore mined on the Plateaus was oxidized vanadium-uranium ore from the western Colorado-eastern Utah area, now known as the Uravan mineral belt, and from Temple Mountain on the east side of the San Rafael swell in Utah. The most abundant uranium mineral was carnotite with a smaller amount of tyuyamunite and very little rauvite and uvanite. The most abundant vanadium minerals (aside from the uranyl vanadates) were vanadium hydromica and/or roscoelite and corvusite, with • local concentration of hewettite and metahewettite and small amounts of other secondary quinquivalent vanadium minerals: pascoite, hummerite, rossite, metarossite, steigerite, navahoite, fervanite, and the sodium analogue of hewettite--filling fractures or coating joint surfaces and mine walls。

Many of these minerals have been found in mines recently developed at Monument Valley, Ariz. In the Shiprock district, Arizons-New Mexico, and along the north side of the Zuni uplift, No Mex., tyuyamunite and metatyuyamunite are more abundant than carnotite. Recently several other uranium minerals in small amounts have been found in carnotite ore: schroeckingerite, meta-autunite, metazeunerite, uranophane, and novacekite. Locally, where both copper and vanædium are present, small quantities of volborthite and calciovolborthite occur as at Richardson Basin, Moab district, Utah, and in the Slick Rock district, Colo. 
Placerville and Rifle, Colo.--two areas that produced chiefly vanadium with relatively little uranium--have not been given detailed mineralogic study.

The vanadium-to-uranium ratio of the ores ranges from a high ratio of about 30:1 at Placerville and Rifle, Colo., through lower values in the Uravan mineral beit, Colorado-Utah, the Shiprock district, Arizona-New Mexico, Monument Valley, Ariz., the Grants district on the north side of the Zuni uplift, N. Mex., to a ratio of about $1: 1$ at Temple Mountain in the San Rafael district, Utah. Some differences in relative abundance of minerals in the several areas are due to the variation in $\mathrm{V}: U$ ratio. Other differences are due to local conditions such as high calcium content of the sediments in the Shiprock and Grants districts causing local predominance of tyruamunite. In the Uravan belt the predominance of carnotite seems to be coincident with the area of the Pennsylvanian evaporite basin and may be related to the presence of potassium salts in the Paradox member of the Hermosa formation. Presence of fossil bone may favor local development of autunite. In the Grants district the fluorite associated with ore may be related to fluorite deposits in the center of the Zuni uplift.

Unoxidized vanadium-uranium ore

In the early days of uranium mining on the Plateaus, small concentrations of black minerals included in the carnotite ore were called corvusite-vanoxite ore and thought to be composed chiefly of vanadium oxides.

Recently, as many new mines have been opened, much more black ore high in uranium as well as vanadium has been found wherever ore bodies are protected from oxidation by thick cover, as in the deeper ore bodies in the Long Park area of the Uravan district, or where ore has been exposed very recently 
by headward erosion of steep canyons, as in Lumsden Canyon, Gateway district, or La Sal Creek, Paradox district. Some small mines have chiefly black ore with very little secondary alteration, and others like Monument No. 2 mine in Monument Valley district, have scattered unoxidized remnants in ore that is chiefly oxidized.

Since 1950 mineralogic study has shown the presence of several important primary uranium and vanadium minerg.1s. The uranium minerals are pitehblende* (identified in 11 mines of uranium-vanadium ore) and a new uranium mineral (identified in 8 mines of uranium-vanadium ore). The new mineral is callied coffinite by L. R. Stieff and T. W. Stern who found it in 1951 (report in preparation). The vanadium minergls are montraseite and other trivalent and quadrivalent vanadium oxides (one called doloresite and another Iumsdenite by T.W. Stern, report in preparation). Both uranium and vanadium minerals are associated with pyrite, comonly with high rank coalified wood, and traces of copper, lead, cobalt, nickel, molybdemu, and silver. Also present and possibly representing a transition to the oxidized ore are melanovansite, corrusite and probably fernandinite.

The ore at Temple Mountain in the eastern part of the San Rafael district has been commonly referred to as asphaltite ore because of asphaltic material impregnating the sandstone and carbonaceous material in the ore. However, higher-than-gverage carbon content does not prevent classifying this ore on the basis of mineral assemblage with the other uranium-vanadium ores. The relatively unoxidized portion of the ore contains pitchblende associated with a hard carbonaceous substance variously described as high rank coal or

*The term pitchblende is used as in Dana, 7 th edition, vol. 1, pp. 613614, for a massive variety of uraninite, with specific gravity lower than. 8.5 and thorium absent or less than 1 percent. 
polymerized petroleum residue (thucholite ?); also present is pyrite with very small amounts of montroseite and galena.

\section{Oxidized nonvanadiferous uranium ores}

In contrast to the carnotite ore, the nonvanadiferous uranium ores are characterized by a wide variety of secondary uranium minerals that include hydrated oxides, carbonates, sulfates, phosphates, arsenates, and silicates. Most of these uranium minerals are yellow, orange, greenish yellow, or green, and microcrystalline or massive. They fill minute fractures in sandstone, conglomerate, or fossil wood and coat joint surfaces and mine walls, In small ore pockets or even in small mines one of these minerals may be abundant, but among them no mineral is as predominant as carnotite is in the oxidized vanadium-uranium ore. In studies to date, the uranium sulfates seem the most abundant.

Copper, the chief associated metal, occurs in many secondary minerals, commonly as malachite, azurite, chalcanthite, antlerite, brochantite, and chrysocolla, and rarely as conichalcite, chalcoalumite, and volborthite. Other metals are present and differ in abundance from one mine to another: iron and manganese in limonite and wad, cobalt in bieberite (comonly dehydrated), sphaerocobaltite, erythrite, or cobaltoan pickeringite, molybdenum in ilsemannite or ferrimolybdite, and traces of lead, zinc, nickel and silver.

Outcrops of these deposits or joint surfaces within a few inches of the cliff face commonly show bright-blue and green copper stain, bright yellow of uranium sulfates or carbonates, pink cobalt bloom, dull-yellow jarosite, wite alunite and, in a few places, fluorescent uraniferous opal and allophane. Clay lenses in the ore or nearby are bleached and altered 
to kaolinite, jarosite, alunite, or gibbsite.

Unoxidized nonvanadiferous ore (pitchblende-copper sulfide)

Since 1949 relatively unoxldized nonvanadiferous uranium ore has been found at a number of places in the west-central part of the Monument uplift, now called the white Canyon mining district, and in scattered localities in the Green River, San Rafael, and Henry Mountains mining districts. Coffinite has been identified at 1 mine and pitchblende at 13 mines in nonvanadiferous ore (in addition to the 8 coffinite and 11 pitchblende localities in vanadiferous ore). As in the case of the black vanadium-uranium ore, some mines have chiefly unoxidized ore and others have unoxidized. remnants in ore that is fairly well oxidized.

The best development of relatively unoxidized nonvanadiferous ore is the pitchblende-copper sulfide deposit in which the Happy Jack mine is located at White Canyon, Utah. The ore contains both sooty pitchblende and massive pitchblende that is so pure and of such high specific gravity (9.0) as to justify calling it uraninite. Some of the pitchblende replaces fossil wood and some, in tabular masses, does not show wood structure. It is closely associated with chalcopyrite, pyrite, bornite, chalcocite, sphalerite, and galena and traces of cobalt, nickel, molybdenum, and silver. The abundance of secondary uranium sulfates as efflorescences on the mine walls shows the close relation between pitchblende and the sulfides. 
Part II

URANIUM MINERAIS

\section{Description of identified minerals}

The uranium minerals described in the following pages are those from , the Colorado Plateaus that the authors have studied. One species, uvanite, exists only in the type specimen in the U. S. National Museum and no new localities have been found.

Classified according to chemical composition the described minerals are:

\begin{tabular}{|c|c|}
\hline Oxides & $\begin{array}{l}\text { Uraninite and pitchblende } \mathrm{UO}_{2} \\
\text { Becquerelite } 2 \mathrm{UO}_{3} \cdot 3 \mathrm{H}_{2} \mathrm{O} \\
\text { Fourmarierite } \mathrm{PbU}_{4} \mathrm{O}_{13} \cdot 7 \mathrm{H}_{2} \mathrm{O}\end{array}$ \\
\hline Carbonates & $\begin{array}{l}\text { Bayleyite } \mathrm{Mg}_{2}\left(\mathrm{UO}_{2}\right)\left(\mathrm{CO}_{3}\right)_{3} \cdot 18 \mathrm{H}_{2} \mathrm{O} \\
\text { Schroeckingerite } \mathrm{NaCa}_{3}\left(\mathrm{UO}_{2}\right)\left(\mathrm{CO}_{3}\right)_{3}\left(\mathrm{SO}_{4}\right) \mathrm{F} \cdot 1 \mathrm{H}_{2} \mathrm{O} \\
\text { Liebigite } \mathrm{Ca}_{2} \mathrm{U}\left(\mathrm{CO}_{3}\right)_{4} \cdot 1 \mathrm{OH}_{2} \mathrm{O}(?) \\
\text { Rabbittite } \mathrm{Ca}_{3} \mathrm{Mg}_{3}\left(\mathrm{UO}_{2}\right)_{2}\left(\mathrm{CO}_{3}\right)_{6}(\mathrm{OH})_{4} \cdot 18 \mathrm{H}_{2} \mathrm{O}\end{array}$ \\
\hline Sulfates & $\begin{array}{l}\text { Uranopilite }\left(\mathrm{UO}_{2}\right)_{6}\left(\mathrm{SO}_{4}\right)(\mathrm{OH})_{10} \cdot 12 \mathrm{H}_{2} \mathrm{O} \\
\text { Johannite } \mathrm{Cu}\left(\mathrm{UO}_{2}\right)_{2}\left(\mathrm{SO}_{4}\right)_{2}(\mathrm{OH})_{2} \cdot 6 \mathrm{H}_{2} \mathrm{O} \\
\text { Betazippeite }\left(\mathrm{UO}_{2}\right)_{2}\left(\mathrm{SO}_{4}\right)(\mathrm{OH})_{2} \cdot 4 \mathrm{H}_{2} \mathrm{O}\end{array}$ \\
\hline Phosphates & $\begin{array}{l}\text { Autunite } \mathrm{Ca}\left(\mathrm{UO}_{2}\right)_{2}\left(\mathrm{PO}_{4}\right)_{2} \cdot 10-12 \mathrm{H}_{2} \mathrm{O} \\
\text { Meta-autunite } \mathrm{I} \quad \mathrm{Ca}_{2}\left(\mathrm{UO}_{2}\right)_{2}\left(\mathrm{PO}_{4}\right)_{2} \cdot 2 \frac{1}{2}-6 \frac{1}{2} \mathrm{H}_{2} \mathrm{O} \\
\text { Bassetite } \mathrm{Fe}\left(\mathrm{UO}_{2}\right)_{2}\left(\mathrm{PO}_{4}\right)_{2} \cdot 8 \mathrm{H}_{2} \mathrm{O} \\
\text { Torbernite } \mathrm{Cu}\left(\mathrm{UO}_{2}\right)_{2}\left(\mathrm{PO}_{4}\right)_{2} \cdot 8-12 \mathrm{H}_{2} \mathrm{O} \\
\text { Metatorbernite } \mathrm{Cu}\left(\mathrm{UO}_{2}\right)_{2}\left(\mathrm{PO}_{4}\right)_{2} \cdot 8 \mathrm{H}_{2} \mathrm{O} \\
\text { Phosphuranylite } \mathrm{Ca}_{3}\left(\mathrm{UO}_{2}\right)_{5}\left(\mathrm{PO}_{4}\right)_{4}(\mathrm{OH})_{4} \cdot 2 \mathrm{H}_{2} \mathrm{O} \text { (?) }\end{array}$ \\
\hline Arsenates & $\begin{array}{l}\text { Metazeunerite } \mathrm{Cu}\left(\mathrm{UO}_{2}\right)_{2}\left(\mathrm{AsO}_{4}\right)_{2} \cdot 8 \mathrm{H}_{2} \mathrm{O} \\
\text { Novacekite } \mathrm{Mg}\left(\mathrm{UO}_{2}\right)_{2}\left(\mathrm{AsO}_{4}\right)_{2} \cdot 8-1 \mathrm{H}_{2} \mathrm{O}\end{array}$ \\
\hline Vanadates & 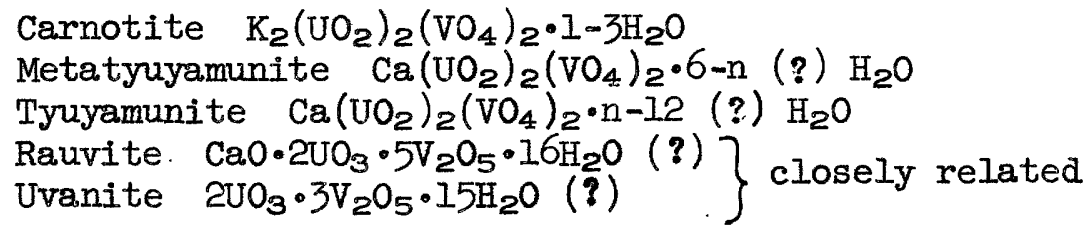 \\
\hline icates & $\begin{array}{l}\text { Uranophane } \mathrm{Ca}\left(\mathrm{UO}_{2}\right)_{2} \mathrm{Si}_{2} \mathrm{O}_{7} \cdot 6 \mathrm{H}_{2} \mathrm{O} \\
\text { Cuprosklodowskite } \mathrm{Cu}\left(\mathrm{UO}_{2}\right)_{2} \mathrm{Si}_{2} \mathrm{O}_{7} \cdot 6 \mathrm{H}_{2} \mathrm{O}\end{array}$ \\
\hline
\end{tabular}


New mineral of uncertain formula

Uranium-bearing materials
Coffinite, black mineral having $X$-ray pattern like thorite.

Organic material, opal, allophane, limonite, and wad.

Additional minerals from the Colorado Plateaus noted in Atomic Energy Commission reports are schoepite $\mathrm{UO}_{3} \cdot 2 \mathrm{H}_{2} \mathrm{O}$, sabugalite $\mathrm{HAI}\left(\mathrm{UO}_{2}\right)_{4}\left(\mathrm{PO}_{4}\right) \cdot 16 \mathrm{H}_{2} \mathrm{O}$, sklodowskite $\mathrm{Mg}\left(\mathrm{UO}_{2}\right)_{2} \mathrm{Si}_{2} \mathrm{O}_{7} \cdot 7 \mathrm{H}_{2} \mathrm{O}$, and beta-uranophane $\mathrm{Ca}\left(\mathrm{UO}_{2}\right)_{2} \mathrm{Si}_{2} \mathrm{O}_{7} \cdot 6 \mathrm{H}_{2} \mathrm{O}$. Three new uranium carbonates, swartzite $\mathrm{CaMg}\left(\mathrm{UO}_{2}\right)\left(\mathrm{CO}_{3}\right)_{3} \cdot 12 \mathrm{H}_{2} \mathrm{O}$, andersonite $\mathrm{Na}_{2} \mathrm{Ca}\left(\mathrm{UO}_{2}\right)\left(\mathrm{CO}_{3}\right)_{3} \cdot 6 \mathrm{H}_{2} \mathrm{O}$, and bayleytte were described from the Hillside mine, Yavepai County, Arlz., (Axelrod and others, 1951). Bayleylte has been found in a second occurrence in a copper-urantum deposit in White Canyon district, Utah (Stern and Weeks, 1952). Soddylte $\left(\mathrm{UO}_{2}\right)_{5}\left(\mathrm{S1O}_{3}\right)_{2}(\mathrm{OH})_{6} \cdot 3 \mathrm{H}_{2} \mathrm{O}$, is known to occur also in Yavapal County, Arlz. (R. Berman, personal comunication). Other minerals that may be found in the Plateaus deposits include uranocircite $\mathrm{Ba}\left(\mathrm{UO}_{2}\right)_{2}\left(\mathrm{PO}_{4}\right)_{2} \cdot 8 \mathrm{H}_{2} \mathrm{O}$, uranospinite $\mathrm{Ca}\left(\mathrm{UO}_{2}\right)_{2}\left(\mathrm{AsO}_{4}\right)_{2} \cdot 8-12 \mathrm{H}_{2} \mathrm{O}$, saléelte $\mathrm{Mg}\left(\mathrm{UO}_{2}\right)_{2}\left(\mathrm{PO}_{4}\right)_{2} \cdot 8-1 \mathrm{OH}_{2} \mathrm{O}$, and sengierite $\mathrm{Cu}\left(\mathrm{UO}_{2}\right)\left(\mathrm{VO}_{4}\right)(\mathrm{OH}) \cdot 4-5 \mathrm{H}_{2} \mathrm{O}$ (?).

Several yellow uranium minerals from the Plateaus are still unidentified and some of these are probably new minerals. When work on these is completed descriptions of the new minerals will be given in a second edition of this report. 
AUTUNITE

and META-AUTUNITE I

$$
\begin{gathered}
\mathrm{Ca}\left(\mathrm{UO}_{2}\right)_{2}\left(\mathrm{PO}_{4}\right)_{2} \cdot 10-12 \mathrm{H}_{2} \mathrm{O} \\
\text { (Meta-autunite I has } \left.2 \frac{1}{2} \cdot 6 \frac{1}{2} \mathrm{H}_{2} \mathrm{O}\right)
\end{gathered}
$$

Crystal system: Tetragonal; ditetragonal-dipyramidal $4 / \mathrm{m} 2 / \mathrm{m} 2 / \mathrm{m}$

Habit: Thin tabular $\{001\}$. As foliated or scaly aggregates.

Physical properties:

Color: ranges from lemon yellow to pale green. Streak yellowish: Fluorescence: strong yellow green. Meta-autunite less strong. Luster: vitreous, pearly on $\{001\}$. Cleavage: $\{001\}$ perfect, $\{100\}$ indistinct. Not brittle. Hardness: $2-2$ it $3.1-3.2$, varying with the water content. Strongest lines of $\mathrm{X}$-ray powder pattern: VS $8.3, \mathrm{~S} 3.59, \mathrm{~W} 1.60$ (Meta $\leadsto$ gutunite I)

Optical properties:

$\begin{array}{ll}\text { Orientation } & \underline{\mathrm{n}} \\ \mathrm{X}=\mathrm{c} & 1.553-? \\ \mathrm{Y} & 1.575-1.59 \\ \mathrm{Z} & 1.577=1.61 \\ \text { 2V usual1y } 10-30^{\circ} ; \mathrm{r}>\mathrm{v} \text { strong }\end{array}$

Pleochroism

Colorless to pale yellow Yellow to dark yellow Yellow to dark yellow Usually anomalously biaxial negative due to loosely held water in both autunite and meta-autunite $I$

Meta-autunite from Thom claim is biaxial negative, $2 \mathrm{~V}$ small to medium, $\mathrm{nY}$ and $\mathrm{nZ}$ equal to $1.603 \pm 0.003$

Analysis: Qualitative spectrographic analysis of material from Thom claim.

$\begin{array}{ll}\text { Major } & \text { U P } \\ \text { Minor } & \text { Ca Si Fe } \\ \text { Trace } & \text { Al Co Na As Ni Mg Pb }\end{array}$

Occurrence and associated minerals: Coating fracture surfaces of weathered brown sandstone.

Identification: On drying or slight heating autunite passes reversibly to meta-autunite I

Optical properties are quite variable. Use with caution. $\mathrm{X}=\mathrm{ray}$ powder pattern. Analysis for $\mathrm{Ca}$ necessary to distinguish from uranocircite.

Locality: Thom claim, Thompson district. 
Crystal system: Monoclinic, pseudo-orthorhombic

Habit: Scaly, flattened on $\{010\}$.

Physical properties:

Color: yellow

Fluorescence: yellow, weak, variable

Luster: pearly Hardness: $\{010\}$ perfect. $\{100\}$ and $\{001\}$ distinct.

Specific gravity: 3.10

Strongest lines of X-ray powder pattern: S $9.4, \mathrm{M} 3.48, \mathrm{M} 2.19$

Optical properties:

Orientation

$X=b$

$Y$

$\mathrm{Z} \wedge \mathrm{c}=40^{\circ}$
$\underline{n}(\mathrm{Na})$

$[\sim 1.56]$

1.574

1.580
Pleochroism

Pale yellow Deep yellow Deep yellow
Biaxial negative $2 V \sim 52^{\circ}$

Analysis: Qualitative spectrographic analysis

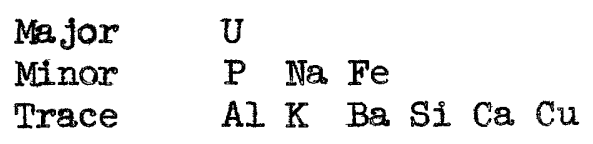

Occurrence and. associated minerals: Secondary coating on mine wall, near the portal.

Identification: X-ray powder pattern.

Locality: Denise No. 1 mine, Green River district. 
BAYLEYITE

$\mathrm{Mg}_{2}\left(\mathrm{UO}_{2}\right)\left(\mathrm{CO}_{3}\right)_{3} \cdot 18 \mathrm{H}_{2} \mathrm{O}$

Crystal form: Monoclinic

Habit: Minute prismatic crystals.

Physical properties:

Color: sulfur yellow

Fluorescence: feeble

Luster: vitreous

Cleavage:

Hardness: $2-2 \frac{1}{2}$

Specific gravity: 2.05

Strongest lines of X-ray powder patterns: $S 7.6$, s 13.0 , M 3.82

Optical properties:

$\begin{array}{llll}\text { Orientation } & \underline{n} & \text { Pleochrolsm } & \\ \mathrm{X} \wedge \mathrm{c} 14^{\circ} & 1.455 & \text { Pinkish } & \\ \mathrm{Y} & 1.490 & \text { Pale yellow } & \text { Biaxial negative } \\ \mathrm{Z}=\mathrm{b} & 1.500 & \text { Pale yellow } & \\ 2 \mathrm{~V}=30^{\circ} & & \end{array}$

Analysis: Semiquantitative spectrographic analysis, in percent, of material from Hideout mine.

$\begin{array}{cl}>10 & \mathrm{U} \\ 1.0-10 & \mathrm{Mg} \mathrm{Ca} \text { (Iow) } \\ 0.1-1.0 & \mathrm{Al} \\ 0.01-0.1 & \mathrm{Si} \mathrm{Sr}\end{array}$

Occurrence and associated minerals: Found with schroeckingerite and gypsum as a coating on mine wall.

Identification:

Soluble in water. Effervesces in $\mathrm{HCl}_{\text {. }}$

Optically distinct. Indices unusua.1ly low for a uranium mineral.

Hideout material did not dehydrate in lab, as Arizona material did.

Localities:

Hillside mine, Yavapai County, Arizona, type locality.

Hideout (Tiger) mine, White Canyon district. 
BECQUEREI,ITE

$$
2 \mathrm{UO}_{3} \cdot 3 \mathrm{H}_{2} \mathrm{O}
$$

Crystal system: Orthorhombic; dipyramidal 2/m 2/m 2/m

Habit: Tabular $\{001\}$ and elongated [010] Also massive.

Physical properties:

Color: amber to brownish yellow. Streak yellow.

Fluorescence:

Luster: adamantine, inclining to greasy.

Cleavage: \{001\} perfect, also $\{101\}$

Hardness: $2-3$

Specific gravity: 5.2

Strongest lines of X

Optical properties:

Orientation

$\underline{\text { Pleochroism }}$

$X=c$

1.735

1.820

Colorless

$Y=b$

1.830

Light yellow

Biaxial negative

$\mathrm{Z}=\mathrm{a}$

$2 \mathrm{~V}=31^{\circ} ; \mathrm{r}>\mathrm{V}$ marked

Dark yellow

Analysis: Qualitative spectrographic analysis of material from Posey mine.

$\begin{array}{ll}\text { Major } & \mathrm{U} \\ \text { Minor } & \mathrm{SI} \\ \text { Trace } & \mathrm{Cu} \mathrm{Fe} \mathrm{Mg}\end{array}$

Occurrence and associated minerals: Alteration product of pitchblende at Cato Sells. At Posey in high-grade pocket of yellow oxidized ore with cuprosklodowskite.

Identification:

X $\rightarrow$ ray powder pattern.

Loca.lities :

Posey mine, White Canyon district

Cato Sells mine, Monument Valley district

Monument No. 2 mine, Monument Valley district. 
CARMOTITE

$$
\mathrm{K}_{2}\left(\mathrm{UO}_{2}\right)_{2}\left(\mathrm{VO}_{4}\right)_{2} \cdot 1-3 \mathrm{H}_{2} \mathrm{O}
$$

Crystal system: Monoclinic

Habit: As a powder or as loosely coherent microcrystalline aggregates, some may be compact; disseminated:; rarely as crusts of imperfectly platy crystals, flattened $\{001\}$ 。

Physical properties:

Colar: lemon yellow, greenish ye11ow: at Pumpkin Buttes orange. Fluorescence: none

Luster: dull or earthy: pearly or silky when coarsely crystalline Cleavage: $\{001\}$ perfect.

Hardness: soft

Specific gravity: $4-5: 4.6$ average of 4 measurements on

crystaline carnotite.

Strongest Iines of $X-r a y$ powder patterns: S 6.5, M 3.11, W 3.51

Optieal properties:

Orientation $\quad \underline{n} \quad$ Pleochroism

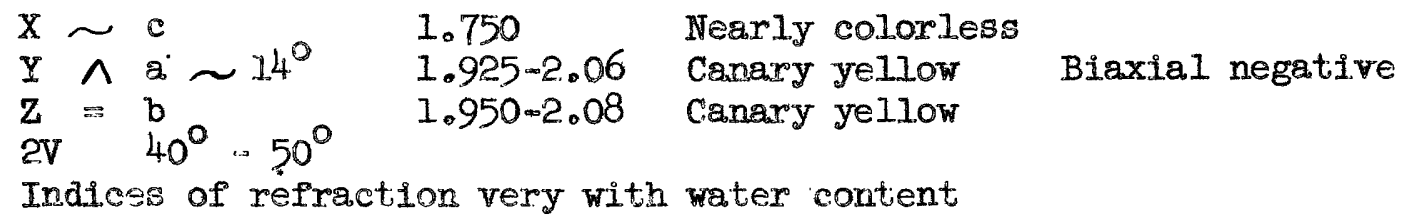

Analysis: Semiquantitative spectrographic analysis, in percent, of material from near Cane Springs Pass, Moab district (USNM 95332)

$$
\begin{aligned}
& >10 \text { U V } \\
& 1=10 \mathrm{~K} \\
& 0.1-1 \text { AI Ca Na Si } \\
& 0.01=0.1 \mathrm{Ba}, \mathrm{Sr} \mathrm{Fe}
\end{aligned}
$$

Occurrence and associated minerals: Chiefly disseminated in sandstone or locally as sma11. pure masses, especial1y around petrified or carbonized tree trunks or other vegetal matter. Associated with tyuyamunite, metatyuyamunite, hewettite, rauvite, and corvusite. At Monument No.2 mine, with pitchblende.

Identification: Carnotite has higher indices of refraction than any other yellow uranium mineral. Carnotite and tyuyamunite turn red-brown when a drop of concentrated $\mathrm{HCl}$ is added. Tyuyamunite fuses relatively easily; carnotite is infusible.

Localities: In most of the vanadium-uranium mines of the following districts on the Colorado Plateaus: Thompsons, Gateway, Uravan, Paradox, Bull Canyon, Gypsum Valley, Slick Rock, Moab, Monticello, Monument Valley, Grants, and the Temple Mountain part of the San Rafael district. Also at Pumpkin Buttes, Wyo., and at Craven Canyon, Fall River County, S. Dak. 
COFFINITE

Formula uncertain*

Crystal system: Tetragonal

Habit: Massive; may show remnants of wood structure.

Physical properties:

Color: black

Fluorescence: none

Luster: adamantine

Cleavage:

Hardness:

Specific gravity: about $3.3-3.5$

Strongest lines of X-ray powder pattern: $\mathrm{S} 3.48$, Ms 4.62, M 2.64, M 1.80

Optical properties: Opaque. Translucent in very thin fragments.

Analysis: No good analysis is yet avallable; mineral occurs mixed with carbonaceous material and other black minerals.

Occurrence and associated minerals: Impregnating sandstone and eplacing wood: with uraninite, and a low valence $(+3,+4)$ vanadium oxide (doloresite), and pyrite. Found in mines with protective cover or at the heads of steep canyons where erosion has recently exposed ore. (Coffinite was first found at Ia Sal No. 2 mine in August 1951 by T. Wo Stern and I. R. Stieff, report in preparation; named for R. C. Coffin.)

Identification: X-ray powder pattern and lack of thorium. The X-ray powder pattern is very similar to that of thorite.

Localities:

Arrowhead mine

Black mine

Corvusite mine

La Sa.I No. 2 mine

Matchless mine
Gateway dist. Gateway dist. Gateway dist. Gateway dist. Gateway dist.
Gray Dawn mine

Wild Steer mine Little Muriel mine Denise No. 1 mine
Paradox dist. Bull Canyon dist. Slick Rock dist. Green River dist.

*Analyses show up to 61 percent $U$ and varying amounts of Si, As, and V. Coffinite may be analogous to thorite $\left(\mathrm{ThSiO}_{4}\right)$, i.e., USiO 4 , with $\mathrm{As}, \mathrm{V}, \mathrm{OH}$, etc., substituting for $\mathrm{Si}$, or it may be a hydrated oxide. (June 1953). 
Crystal system: Orthorhombic

Habit: Minute prismatic or acicular crystals. Usually grouped in radial clusters, also as thin films and botryoidal crusts.

Physical properties:

Color: pale yellow green; yellow in thin crystal blades. Fluorescence:

Luster: pearly to dull

Cleavage: $\{100\}$ and $\{010\}$

Hardness: $3-4$

Specific gravity: $3.5 \pm$

Strongest lines of $\mathrm{X}$-ra⿳亠 powder pattern: VS $8.1, \mathrm{~s} 4.08, \mathrm{M} 6.1$

Optical properties:

\begin{tabular}{lcl} 
Orientation & $\underline{\mathrm{n}}$ & \multicolumn{2}{l}{ Pleochrolsm } \\
$\mathrm{X}$ & 1.654 & Very pale yellowish green \\
$\mathrm{Y}$ & $1.664-1.667$ & Very pale yellowish green \\
$\mathrm{Z}=\mathrm{c}$ & $1.664-1.667$ & Pale greenish yellow \\
2V sma1l: $\mathrm{r}>\mathrm{v}$ strong & &
\end{tabular}

Analysis: Qualitative spectrographic analysis of material from Posey mine.

$$
\begin{array}{ll}
\text { Major } & \mathrm{U} \text { Si } \\
\text { Minor } & \mathrm{Cu} \\
\text { Trace } & \mathrm{Pb} \text { Fe Na }
\end{array}
$$

Occurrence and associated minerals: As a fracture coating with brochantite. In a high-grade pocket as thin green veins in massive becquerelite.

Identification: X-ray powder pattern, or spectrographic analysis of a pure sample.

Localities:

Posey mine, White Canyon district 
Crystal system: Orthorhombic

Habit: Tabular $\{001\}$ and usually elongated [010]

Physical properties:

Color: red to golden red; also brown

Fluorescence:

Luster: adamantine

Cleavage: $\{001\}$ perfect

Hardness: $3-4$

Specific gravity: 6.0

Strongest lines of X-ray powder pattern: $s 3.38,53.04,51.89$

Optical properties:

$\begin{array}{llll}\text { Orientation } & \underline{n} & \text { Pleochroism } & \\ \mathrm{X}=\mathrm{c} & 1.85 & \text { Colorless } & \\ \mathrm{Y}=\mathrm{b} & 1.92 & \text { Pale yellow } & \text { Biaxial negative } \\ \mathrm{Z}=\mathrm{a} & 1.94 & \text { Yellow } & \\ \text { 2V large; } \mathrm{r}>\mathrm{v} \text { strong } & & \end{array}$

Analysis: Qualitative spectrographic analysis of material from Lucky Strike No. 2 mine

$$
\begin{array}{ll}
\text { Major } & \mathrm{U} \\
\text { Minor } & \mathrm{Pb} \\
\text { Trace } & \mathrm{Al} \mathrm{Mg} \mathrm{Si} \mathrm{Fe}
\end{array}
$$

Occurrence and associated minerals: As an alteration product of pitchblende, with beta-zippeite.

Identification: Orange-red color and test showing more than 10 percent $\mathrm{Pb}$. X-ray powder pattern.

Localities :

Lucky Strike No. 2 mine, San Rafael district Monument No. 2 mine, Monument Valley district 
JOHANNITE

$$
\mathrm{Cu}\left(\mathrm{UO}_{2}\right)_{2}\left(\mathrm{SO}_{4}\right)_{2}(\mathrm{OH})_{2} \cdot 6 \mathrm{H}_{2} \mathrm{O}
$$

Crystal system: Triclinic; pinacoidal I

Habit: Prismatic; as coatings and small spheroidal aggregates of lath-like crystals.

Physical properties:

Color: clear, light green. Streak pa.ler. Fluorescence: none

Luster: vitreous

Cleavage: $\{100\}$ good. Not brittle

Hardness: $2-2 \frac{1}{2}$

Specific gravity: 3.32

Strongest lines of X-ray powder pattern: S $7.8, \mathrm{~S} 6.2, \mathrm{M} 3.88$.

Optical properties:

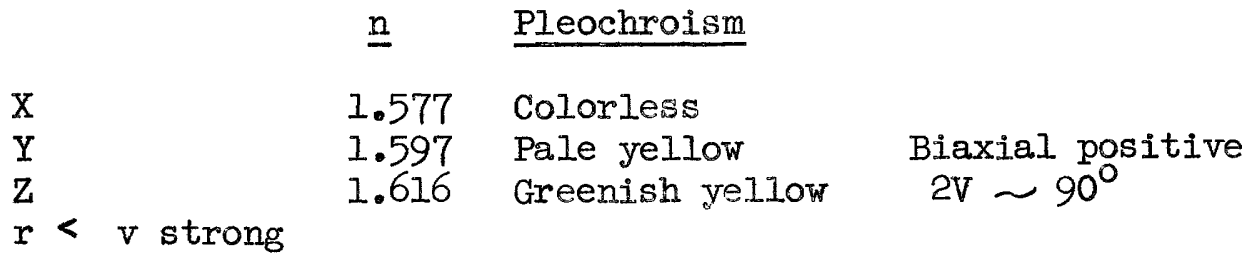

Analysis: Qualitative spectrographic analysis of material from Happy Jack mine.

$\begin{array}{ll}\text { Major } & \mathrm{U} \\ \text { Minor } & \mathrm{Cu} \\ \text { Trace } & \mathrm{Ca} \text { Al Mg Si }\end{array}$

Occurrence and associated minerals: As wall or fracture coatings with uranopilite, betazippeite, brochantite, and chalcanthite. Coating pitchblende, chalcopyrite, and covellite.

Identification: Color, and tests for $\mathrm{Cu}$ and sulfate. $\mathrm{X}$-ray powder pattern.

Localities :

Happy Jack mine, White Canyon district. Oyler mine, Henry Mountains district. Frey No. 4 mine, White Canyon distriet. 
Crystal system: Orthorhombic

Habit: Crystals equant or short prismatic [00l], usually indistinct with rounded edges. Commonly as granular or scaly aggregates and thin crusts; also botryoidal.

Physical properties:

Color: light greenish yellow

Fluorescence: bright light green

Luster: vitreous, slightly pearly on the cleavage

Cleavage: $\{100\}$

Hardness: $2 \frac{1}{2}-3$

Specific gravity: 2.41

Strongest lines of X-ray powder pattern: S $8.7, \mathrm{~s} 6.8, \mathrm{~s} 5.4$ Effervesces in $\mathrm{HCl}$

Optical properties:

$\begin{array}{llll}\text { Orientation } & \underline{\mathrm{n}} & \text { Pleochroism } \\ \mathrm{X}=\mathrm{a} & 1.497 & \text { Nearly colorless } & \\ \mathrm{Y} & 1.502 & \text { Pale yellowish green } & \text { Biaxial positive } \\ \mathrm{Z} & 1.539 & \text { Pale yellowish green } & \\ \text { 2V } 40^{\circ}, \mathrm{r}>\mathrm{v} \text { moderate } & \end{array}$

Analysis: Qualitative spectrographic analysis of material from

Pumpkin Buttes

$$
\begin{array}{ll}
\text { Major } & \text { U Ca } \\
\text { Minor } & -\infty \\
\text { Trace } & \text { AI Fe Mg Mn Si }
\end{array}
$$

Occurrence and associated minerals:

Secondary coating at Pumpkin Buttes。 Noted by D'Arcy George at Lusk, Wyo., perhaps as alteration product of uranophane (George, 1949).

Identification:

Optical properties. (Be careful to distinguish from bayleyite.).

Localities :

Pumpkin Buttes, Wyo. Lusk, Wyo.

*The valence state of $U$ in this mineral is being checked (U.S.G.S.) 
Crystal system: Tetragonal (or pseudotetragonal)

Hiabit: Thin tabular $\{001\}$. As follated or scaly aggregates.

Pbysical propertie:

Color: straw yellow

Fluorescence: psie yellow green

Luster: pearly

Clearage: $\{001\}$ perfect

Hardness: $2 \frac{1}{2}$

Specific grovity: 3.3

Strongest lines of X-ray powder pattern: VS 10.2, S 3.56, M 5.1

Optical properties:

Orientation

$\underline{\mathrm{n}}$

$X=c$

$Y \quad 1.620-1.623$

$\mathrm{Z} \quad 1.620 \mathrm{~m}-1.623$

Biaxial negative

$2 \mathrm{~V} 0-15^{\circ}$

Analysis: Semiquantitative spectrographic analysis, in percent, of material from Laguna, Grants district.

$$
\begin{array}{cl}
\text { Over } 10 & \text { U Si } \\
1 .-10 & \text { As AI Fe } \\
0.1-i & \text { Mg Ca } \\
0.01-0.1 & \text { TI Sr Ba }
\end{array}
$$

Qecurrence and associated minerals:

Coating on sandstone

Identification:

Color, fluorescence, and test for arsenate distinguish from a.11

but uranospinite $\left[\mathrm{Ca}\left(\mathrm{UO}_{2}\right)_{2}\left(\mathrm{AsO}_{4}\right)_{2} \cdot 8 \cdot-12 \mathrm{H}_{2} \mathrm{O}\right]$

$\mathrm{X}$-ray powder pattern.

Iocalities:

Laguna, Grants district.

This is the only known occurrence in North America, second in world.

(found by T. W. Stern, 1952). 
Crystal system: Tetragonal or pseudo-tetragonal

Habit: As earthy or scaly coatings or crusts, also as micrascopic rectangular plates and laths.

Physical properties:

Color: deep yellow to golden yellow

Fluorescence:

Luster: pearly

Cleavage: $\{001\}$ perfect but not easily observed.

Hardness : $2 \frac{1}{2}$

Specific gravity:

Stronge it lirés of X-ray powder pattern: S 7.9, M 5.83, M 3.92, M.2.88

Oftical properties: variable

n Fleochroism

$\mathrm{X}$ or $\mathrm{E} \quad 1.660-1.690$ Colorless to pale yellow

$\mathrm{Y}$ or $0 \quad 1.700-1.718$ Golden yellow 18 Usually biaxial negative

2V usually $5^{8} \cdot 20^{\circ}$ (up to $35^{\circ}$ ) $x>\mathrm{v}$ strong

Analysis: Good analyses lacking due to occurrence admixed with clay.

Occurrence and associated minerals:

Disseminated in sandstone or as coating on fracture.

Id.entification:

X-ray powder pattern. The optical properties are variable and the mineral is usually too fine grained to exhibit a typical crystal form.

Lacalities:

North Point - Gonway claim, White Canyon district Posey mine, White Canyon district

Cobalt No. 2 mine, Thompsons district

Cactus Rat mine, Thompsons district 
Crystal system: Monoclinic

Habit: Fibrous or finely acicular, in clusters of microscopic crystals: elongated. [001].

Physical properties:

Color: pale greenish yellow

Fluorescence: weak

Luster: silky

cleavage: $\{001\}$

Hardness: soft

Specific gravity: approx.2.5

Strongest lines of X-ray powder pattern: S 8.1, M 11.1, M 4.37

Optical properties:

Orientation

$\mathrm{X} \quad 1.502+0.005$

$Y=b$

$\mathrm{z} \wedge \mathrm{c} \sim 15^{\circ}$

$\underline{\underline{n}}$

$1.508+0.005$

$1.525+0.003$

Biaxial positive (?)

2V large

Aralysis:

Chemical analysis of material from Iucky Strike No. 2 mine (in percent). Analyst: A. M. Sherwood

$\begin{array}{llllll}\mathrm{CaO} & \mathrm{MgO} & \mathrm{UO}_{3} & \mathrm{CO}_{2} & \mathrm{H}_{2} \mathrm{O} & \text { Total } \\ 10.6 & 9.2 & 37.4 & 17.8 & 24.5 & 99.5\end{array}$

Occurrence and associated minerals: Efflorescent coating on mine wall near portal; with gypsum, sphaerocobaltite, bieberite and uranium sulfates.

Idlentification: Habit, optical properties.

Locality: Lucky Strike No. 2 mine, San Rafael district, Uth

Specimen collected by M. E. Thompson. Named for John C. Rabbitt, Chief, Trace Elements Section, U.S.G.S., 1947-1953. 
RAIJVITE

$\mathrm{CaO} \cdot 2 \mathrm{UO}_{3} \cdot 5 \mathrm{~V}_{2} \mathrm{O}_{5} \cdot 16 \mathrm{H}_{2} \mathrm{O}(?)$

Crystal system:

Habit: As dense slickensided masses, botryoidal crusts, and filmy coatings commonly showing shrinkage cracks.

Physical properoties:

Color: brownish red to purplish black. Sometimes dirty orange yellow, streak yellow brown.

Fluorescence: none

Luster: adamantine to waxy. Variable.

Cleavage: none. Brititie

Hardness: soft

Specific gravity: 2.92 (for analyzed material, Monument No. 2 mine)

Strongest lines of X-ray powder pattern: VS 10.5, M (broad) 2.95,

M 3.48, M 3.35.

Optical properties: variable

minutely crystalline

$$
\mathrm{n}=1.89-1.95
$$

Biaxial negative (?)

Analysis: Chemical analysis, in percent, of material from Monument No. 2 mine. ADW $9-51$ (A. M. Sherwood, analyst)

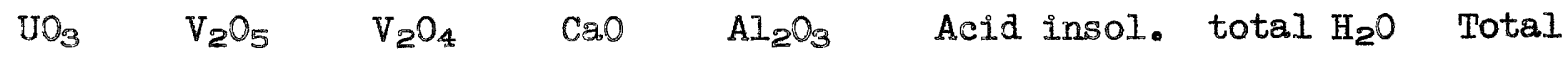

$\begin{array}{llllllll}31.49 & 48.28 & 1.44 & 2.76 & 0.70 & 0.61 & 15.49 & 100.77\end{array}$

Occurrence and associated minera.1s:

Probably an alteration product of pitchblende and low valence vanadium oxides; g.lso possibly of tyuyamunite. Association and occurrence as for tyuyamunite; not as common as tyuyamunite.

Identification:

$X-r a y$ powder pattern. Rauvite is vexy fine grained, and extremely variable in physical properoties.

Localities:

Corvusite mine, Gateway district

Small spot mine, Gateway district

Monument No. 2 mine, Monument Va.IIey district

Temple Mountain, San Rafael district

Arrowhead mine, Gateway district

Cactus Rat mine, Thompsons dictrict 
SCIROECKINGERITE

$$
\mathrm{NaCa}_{3}\left(\mathrm{UO}_{2}\right)\left(\mathrm{CO}_{3}\right)_{3}\left(\mathrm{SO}_{4}\right) \mathrm{F} \cdot 1 \mathrm{OH}_{2} \mathrm{O}
$$

Crystal system: Hexagonal ?

Habit: As clusters or globular aggregates of scales flattened $\{0001\}$, some with a six-sided outline.

Physical properties:

Color: greenish yellow

Fluorescence: strong, greenish yellow

Luster: weakly vitreous, sometimes pearly on (000l)

Cleavage: $\{0001\}$ perfect

Hardness: $2 \frac{1}{2}$

Specific gravity: 2.51

Strongest lines of X-ray powder pattern: S 7.2, M 4.79, M 2.86 .

Soluble in water, effervesces in $\mathrm{HCl}$.

Optical properties:

$$
\underline{\mathrm{n}}
$$

$0 \quad 1.542$

$\mathrm{E} \quad 1.489$

Uniaxial (?) negative

Usually biaxial with small and variable $2 \mathrm{~V}, 0-25^{\circ}$

Analysis: Semiquantitative spectrographic analysis, in percent, of material from Red Desert, Wyo.

$$
\begin{aligned}
& \text { Over } 10 \quad \mathrm{U} \quad \mathrm{Ca} \\
& 0.1-1 \quad F \quad \mathrm{Na} \\
& \text { 0.01-0.1 Si Al Sr Zn Fe } \\
& 0.001-0.01 \quad \mathrm{~K} \text { Ti Mg }
\end{aligned}
$$

Occurrence and associated minerals:

In a near surface deposit in clay at McCoy group, Thompsons district. As coating on mine wall, with bayleyite, at Hideout mine. As alteration product of pitchblende at Crabapple claim.

Identification: X-ray powder pattern. If coarsely crystalline, six-sided plates distinguish it from the other carbonates.

\section{Localities:}

McCoy group, Thompsons district. Crabapple claim, Green River district Hideout (Tiger) mine, White Canyon district 
TORBERNITE

and METATORBERNITE
$\mathrm{Cu}\left(\mathrm{UO}_{2}\right)_{2}\left(\mathrm{PO}_{4}\right)_{2} \cdot 8-12 \mathrm{H}_{2} \mathrm{O}$

Metatorbernite has $8 \mathrm{H}_{2} \mathrm{O}$

(Metatorbernite probably more abundant in nature than torbernite)

Crystal system: Tetragonal; ditetragonal-dipyramidal $4 / \mathrm{m} 2 / \mathrm{m} 2 / \mathrm{m}$

Habit: Tablets on $\{001\}$; often in rosettes or sheaf-like aggregates of irregularly curved and composite crystals.

Physical properties:

Color: pale green to dark green

Fluorescence: not commonly

Luster: vitreous to subadamantine; pearly on $\{001\}$

Cleavage: 001 perfect. Rather brittle

Hardness: $2 \frac{1}{2}$

Specific gravity: $3.5-3.7 \quad$ Torbernite $=3.2$

Strongest lines of X-ray powder pattern-Metatorbernite: VS 8.7 ,

VS 3.68, M 4.93, M 3.49, M 3.35

Optical properties:

Metatorbernite

Torbernite

$\underline{n}$

$0 \quad 1.610-1.628$

in white light

$\mathrm{E}$

Uniaxial positive (?)

Anom. inter. colors $\underline{n} \quad$ Dichroism

$1.592 \quad$ Sky blue

$1.582 \quad$ Green

Analysis: Qualitative spectrographic analysis of material from Markey No. 3 mine

$$
\begin{array}{ll}
\text { Major } & \mathrm{U} \\
\text { Minor } & \mathrm{Cu} \text { Na Si } \mathrm{P} \\
\text { Trace } & \mathrm{Ca} \text { Mg As } \mathrm{Fe}
\end{array}
$$

Occurrence and associated minerals: Crystalline aggregates on sandstone with metazeunerite, pyrite, chalcopyrite, chalcanthite, and alunite.

Identification: Color, crystal form, and absence of arsenic.

Locality: Markey No. 3 mine, White Canyon district 
TYUYAMUNITE

$$
\begin{gathered}
\mathrm{Ca}\left(\mathrm{UO}_{2}\right)_{2}\left(\mathrm{VO}_{4}\right)_{2} \cdot \mathrm{nH}_{2} \mathrm{O} \\
\mathrm{n}=?-12
\end{gathered}
$$

Crystal system: Orthorhombic

Habit: As scales and laths flatened $\{001\}$ and elongated $[100]$; as radial aggregates. Commonly massive, compact to cryptocrystalline; also pulverulent.

Physical properties:

Color: yellow, greenish yellow Fluorescence: none Luster: of crystals adamantine, pearly on $\{001\}$, massive material waxy. Cleavage: $\{001\}$ perfect, micaceous. $\{010\}$ and $\{100\}$ distinct. Hardness: about 2 Specific gravity: 3.62 on fully hydrated material Strongest lines of X-ray powder pattern*: S 9.9, M 4.93, M 3.29, M 3.16

Optical properties:

Orientation $\quad \underline{n} \quad$ Pleochroism

$$
\begin{array}{llll}
X=c & 1.57 \text { calc. } & \text { Nearly colorless } & \\
Y=b & 1.805 \pm .002 & \text { Pale canary yellow } & \text { Biaxial negative } \\
Z=a & 1.851 \pm .002 & \text { Canary yellow } & \\
2 V & 42^{\circ} r<v & &
\end{array}
$$

Analysis: Chemical analysis of material from Small Spot mine, Gateway district, Analyst: R. G. Milkey

$$
\begin{array}{lccccc}
\mathrm{CaO} & \mathrm{UO}_{3} & \mathrm{~V}_{2} \mathrm{O}_{4} & \mathrm{~V}_{2} \mathrm{O}_{5} & \mathrm{H}_{2} \mathrm{O} & \text { Total } \\
6.03 & 57.08 & 0.55 & 20.31 & 16.03 & 100.00
\end{array}
$$
sample.

Recalculated to 100 percent, after $\mathrm{H}_{2} \mathrm{O}$ determination on fully hydrated

Occurrence and associated minerals:

Disseminated in sandstone. Coating joints and fractures, with metatyuyamunite, carnotite, rauvite, corvusite, and hewettite. At Mesa No. 1 mine, Shiprock district, with melanovanadite.

Identification: Tyuyamunite and carnotite can be distinguished from other yellow U-minerals by the presence of vanadium; they will turn red.brown when a drop of concentrated HCl is touched to the mineral. X-ray powder pattern is usually necessary to distinguish from carnotite. When coarsely crystalline may be distinguished optically. Fuses much more easily than carnotite.

Localities: Same as for carnotite. Abundant in Grants and Shiprock districts, with little carnotite.

*Note: Too vigorous grinding of tyuyamunite for a powder pattern destroys 
Crystal system: Orthorhombic

:

Habit: Same as tyuyamunite

Physfcal' properties:

Color: yellow, greenish yellow

Fluorescence: none

Luster: adamantine to pearly

Cleavage: $\{001\}$ perfect, micaceous. $\{010\}$ and $\{100\}$ distinct. Hardness: about 2

Specific gravity: $3.81-3.93$

3 Strongest lines of X-ray powder patterns: . S 8.4, M 4.21, M 3.24, M 3.04

Optifal properties:

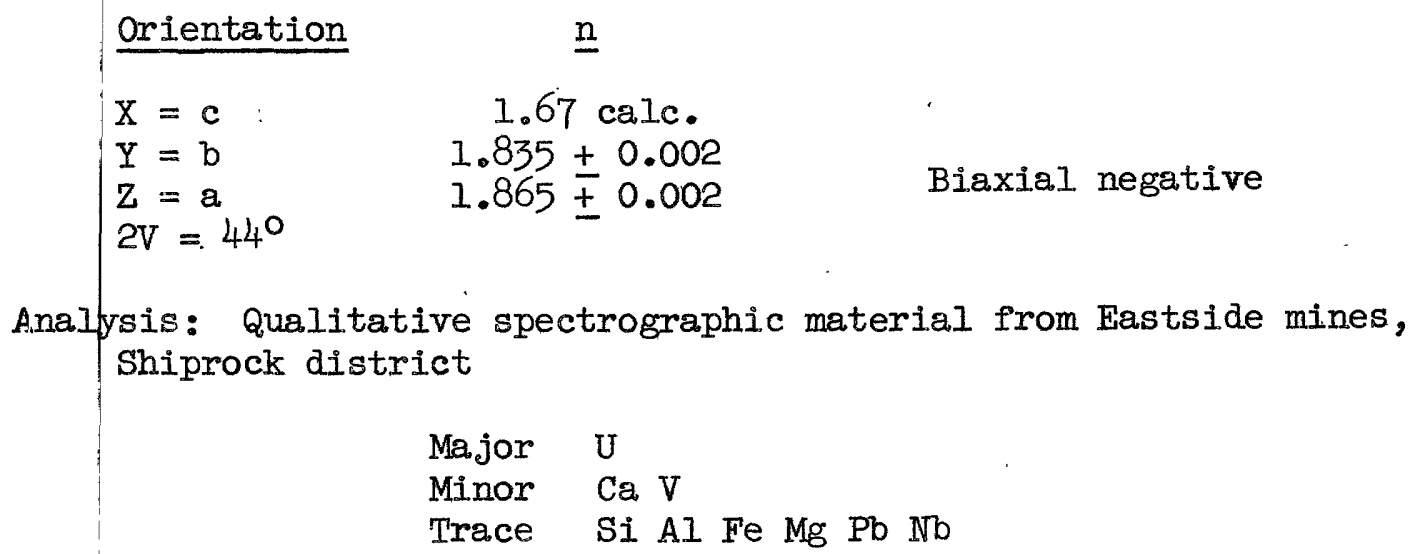

Occurrence and associated minerals:

Same as for tyuyamunite. A dehydration product of tyuyamunite, found at or near surface deposits.

J.dentification:

X-ray powder pattern

Iocalities :

Same as for tyuyamunite.

Especially abundant near Haystack Mountain and Laguna, Grants district. 
URANINITE (Pitchblende)

$$
\begin{aligned}
& \text { Ideally } \mathrm{UO}_{2} \\
& \text { (commonly contains } \mathrm{UO}_{3} \text { ) }
\end{aligned}
$$

Crystal system: Isometric; hexoctahedral $4 / \mathrm{m} \overline{3} 2 / \mathrm{m}$ (?)

Habit: Massive. Commonly replaces cellular structure of wood.

Physical properties:

Color: black

Fluorescence: none

Luster: submetallic to pitchlike or greasy, and dull.

Cleavage: fracture uneven to conchoidal. Brittle. Hardness: $5-6$

Specific gravity: Uraninite 8-10. Colloform pitchblende 48.5

Strongest lines of X-ray powder pattern: VS 3.14 , S 1.65 , S 1.93

Optical properties: Usually opaque. Transparent in very thin splinters.

Anailysis: Qualitative spectrographic analysis of pitchblende from Juniper claim.

$$
\begin{array}{ll}
\text { Major } & \mathrm{U} \\
\text { Minor } & \mathrm{Si} \mathrm{Ca} \\
\text { Trace } & \mathrm{V} \quad \mathrm{Fe} \mathrm{Na} \mathrm{Mn}
\end{array}
$$

Spectrographic analysis of uraninite from Happy Jack shows no element. except uranium over 1 percent.

Occurrence and associated minerals: In unoxidized ore in mines located at the heads of steep canyons or under a protective cover. In vanadiferous ore associated with coffinite and low valence vanadium oxides, montroseite, doloresite, etc., and alters to rauvite, carnotite and tyuyamunite and rarely to becquerelite and uranophane. In non-vanadiferous ores, as at Happy Jack mine, with sulfịdes of $\mathrm{Fe}, \mathrm{Cu}, \mathrm{Pb}, \mathrm{Zn}$, Co, and Ni. Alters to becquerelite, fourmarierite, uranopilite, johannite, betazippeite, schroeckingerite and uranophane.

Iden'tification:

Black, heavy, very radioactive, commonly with yellow alteration products. $\mathrm{X}$-ray powder pattern. 
Uraninite (continued):

Localities :

Morrison formation

Grey Dawn mine

Juniper mine

Corvusite mine

Blue Jay claim
Paradox district Thompson district Gateway district Moab district

Shinarump conglomerate

Camp Bird No. 13 mine Lucky Strike No. 2 mine Marshbank Canyon mine Pay Day mine Rex No. 2 mine Crabapple claim Shinarump No. 1 mine Oyler mine Frey No. 4 mine Happy Jack mine Hideout mine Markey No. 3 mine Notch mine White Canyon No. 1 mine Cato Sells mine Monument No. 2 mine Skyline mine

Others

Haystack Mountain area Placerville, Colo. Huskon No. 2 claim
San Rafael district San Rafael district San Rafael district San Rafael district San Rafael district Green River district Green River district Henry Mountains district White Canyon district White Canyon district White Canyon district White Canyon district White Canyon district White Canyon district Monument Valley district Monument Valley district Monument Valley district

Grants district Placerville district Little Colorado district 
URANOPHANE

$$
\mathrm{Ca}\left(\mathrm{UO}_{2}\right)_{2} \mathrm{Si}_{2} \mathrm{O}_{7} \cdot 6 \mathrm{H}_{2} \mathrm{O}
$$

Crystal system: Orthorhombic

Habit: Minute prismatic in radiated or stellate aggregates. Commonly massive and very finely fibrous.

Physical properties:

Color: yellow, orange yellow, streak paler

Fluorescence: none

Luster: pearly to greasy

Cleavage: $\{100\}$

Hardness: $2-3$

Specific gravity: $3.8-3.9$

Strongest lines of X-ray powder pattern: $s 7.9, \mathrm{~s} 3.95, \mathrm{M} 4.82$, M 2.98, M 2.92

Optical properties:

\begin{tabular}{|c|c|c|c|}
\hline Orientation & $\underline{\mathrm{n}}$ & Pleochroism & \\
\hline$x=a$ & $\begin{array}{l}1.642- \\
1.645\end{array}$ & Colorless & \\
\hline$Y=b$ & $\begin{array}{l}1.6650 \\
1.667\end{array}$ & Pale canary yellow & Biaxia. negative \\
\hline & $\begin{array}{l}1.667- \\
1.670\end{array}$ & Canary yellow & \\
\hline
\end{tabular}

Analysis: Partial chemical analysis by A. Mo Sherwood, TWC-1263, material from Lusk, Wyo.
$\mathrm{H}_{2} \mathrm{O}$
$\mathrm{SiO}_{2}$
$\mathrm{CaO}$
$\mathrm{UO}_{3}$
65.24
Tota1
99.45

occurrence and associated minerals: Disseminated in sandstone at Pumpkin Buttes. At Grants coating limestone. At Cato Sells on pitchblende with becquerelite.

Identification: Index of refraction and absence of $\mathrm{Cu}$ distinguish it from cuprosklodowskite.

Localities: Grants district; Cato Sells mine, Monument Valley district. Pumpkin Buttes and Lusk, Wyo. 
URANOPILITE

$$
\left(\mathrm{UO}_{2}\right)_{6}\left(\mathrm{SO}_{4}\right)(\mathrm{OH})_{10} \cdot 12 \mathrm{H}_{2} \mathrm{O}
$$

Crystal system: Probably monoclinic

Habit: As velvety incrustations and globular or reniform masses composed of microscopic needles or laths elongated [001] and flattened $\{010\}$

Physical properties:

Color: bright yellow

Fluorescence: bright yellow green

Luster: silky

Cleavage: $\{010\}$ perfect

Hardness:

Specific gravity: $3.7-4.0$

Strongest lines of X-ray powder pattern: S 7.1, S 9.1, S 4.23

Optical properties:

Orientation

$\mathrm{X}$

$\mathrm{Y} \wedge \mathrm{c}=18^{\circ}$

$\mathrm{z}$.

2V rather large $(\mathrm{Na}) ; 0^{\circ}$ for some wavelengths

$r<v$ extreme; also $r>v$ $\underline{\mathrm{n}}$

1.623

1.625

1.625 Biaxial positive

Analysis: Qualitative spectrographic analysis of material from Happy Jack mine

$$
\begin{array}{ll}
\text { Major } & \mathrm{U} \\
\text { Minor } & -- \\
\text { Trace } & \text { Si Ca Co }
\end{array}
$$

Occurrence and associated minerals: Abundant in Happy Jack mine as wa.l工 coatings with johannite and betazippeite.

Identification: May be distinguished from zippeite and betazippeite by optical properties. From johannite by test for $\mathrm{Cu}$.

Localities: Happy Jack mine, White Canyon district. 
UVANITE*

$$
2 \mathrm{UO}_{3} \cdot 3 \mathrm{~V}_{2} \mathrm{O}_{5} \cdot 15 \mathrm{H}_{2} \mathrm{O}(?)
$$

Crystal system: Probably orthorhombic

Habit: As minutely crystalline masses and coatings.

Physical properties:

Color: brownish yellow

Fluorescence: none

Luster:

Cleavage: 2 pinacoidal cleavages

Hardness:

Specific gravity:

Strongest lines of X-ray powder pattern: S 2.96, Mb 5.9, Mb 5.3, M 1.71

Optical properties:

$\begin{array}{llll} & \underline{\mathrm{n}} & \text { Pleochroism } & \\ \mathbf{X} & 1.817 & \text { Light brown } & \\ \mathrm{Y} & 1.879 & \text { Dark brown } & \text { Biaxial positive } \\ \mathrm{Z} & 2.057 & & \text { Greenish yellow }\end{array}$

Analysis: W. T. Schaller, Analyst

$\begin{array}{lccccc}\mathrm{CaO} & \mathrm{UO}_{3} & \mathrm{~V}_{2} \mathrm{O}_{5} & \mathrm{H}_{2} \mathrm{O} & \text { Rem. } & \text { Total } \\ 1.73 & 39.60 & 37.70 & 18.28 & 1.69 & 99.00\end{array}$

Occurrence and associated minerals: Associated with carnotite, rauvite, hewettite, metatorbernite, hyalite and gypsum in asphaltic sandstone at Temple Mountain, San Rafael district.

Identification: X-ray powder pattern (3). May be related to rauvite as an alteration product of tyuyamunite. Poorly defined mineral. Needs further work.

Locality: Temple Mountain, San Rafael district, Utah.

*Data from Dana system, 7 th ed., vol. 2, p. 1056, except X-ray powder pattern. No new localities found. 
METAZEUUNERITE

$$
\mathrm{Cu}\left(\mathrm{UO}_{2}\right)_{2}\left(\mathrm{AsO}_{4}\right)_{2} \cdot 8 \mathrm{H}_{2} \mathrm{O}
$$

(fully hydrated zeunerite probably rare in nature)

Crystal system: Tetragonal; ditetragonal dipyramidal 4/m 2/m 2/m

Habit: Tabular $\{00 I\}$ and resembling torbernite.

Physical properties:

Color: grass green to emerald green

Fluorescence: yellow green

Luster: vitreous, pearly on $\{001\}$

cleavage: $\{001\}$ perfect. $\{100\}$ distinct. Hardness: $2-2 \frac{1}{2}$

Specific gravity: 3.6

Strongest lines of X-ray powder pattern: S 8.7, s 3.68, M 5.44, M 4.98

Optical properties:

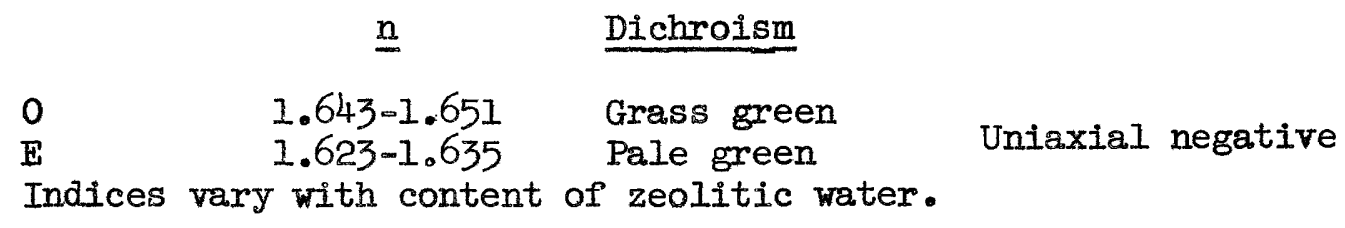

Analysis: Qualitative spectrographic analysis of mineral from Markey No. 3 mine.

$\begin{array}{ll}\text { Major } & \mathrm{U} \\ \text { Minor } & \mathrm{Cu} \text { As } \mathrm{Si} \\ \text { Trace } & \mathrm{Co} \mathrm{Fe} \mathrm{Na} \mathrm{Ca} \mathrm{Pb}\end{array}$

Occurrence and associated minerals: Coating joints and fracture surfaces.

Identification: Test for $\mathrm{Cu}$ and As, with green color, and habit as square plates.

Localities :

Markey No. 3 mine, White Canyon district

Pay Day mine, San Rafael district

Monument Ho. 2 mine, Monument Valley district 
BEYAZIPPEIIE*

$$
\left(\mathrm{UO}_{2}\right)_{2}\left(\mathrm{SO}_{4}\right)(\mathrm{OH})_{2} \cdot 4 \mathrm{H}_{2} \mathrm{O}
$$

Crystal system: Monoclinic (?)

Habit: Microscopic crystalline aggregates; rarely in blades or flakes.

Physical properties:

Color: orange yellow

Fluorescence: green

Iuster: of aggregates dull to $s i l k y$

cleavage: probably $\{010\}$ perfect

Hardness:

Specific gravity: $>3.2$

Btrongest lines of X-ray powder pattern: S 7.1, M 3.13, M 3.49

Opt1cal propertles:

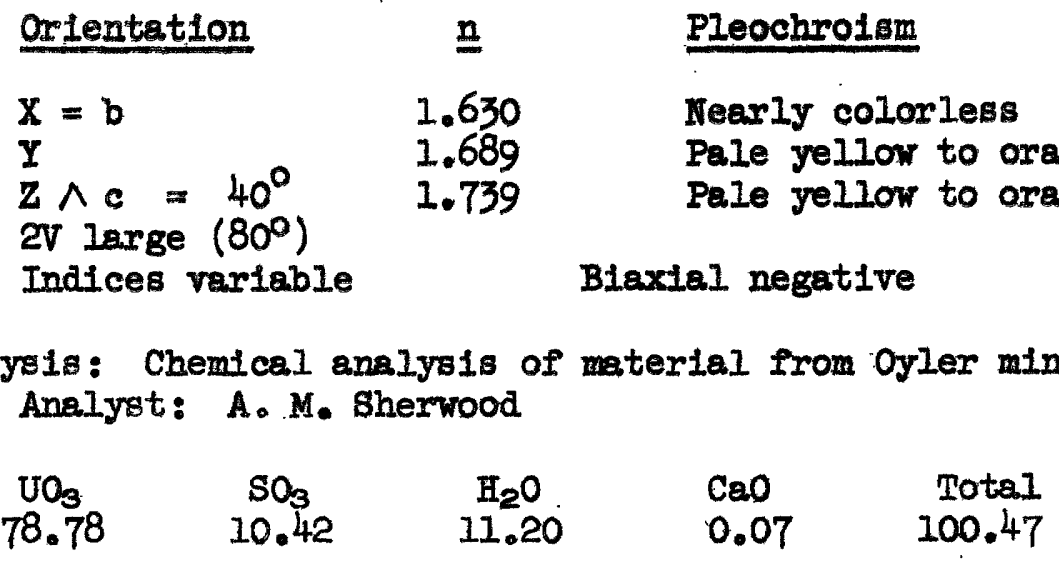

Occurrence and associated minerals: In mines as and fracture coatings. Alone, or with johannite, uranopilite, or pitchblende.

Identification: A sulfate containing no $\mathrm{Cu}$. May be distinguished. optically from uranopilite. Distinguished from zippeite by $X$ aray powder pattern.

Localities:

Happy Jack mine, White Canyon district

Oyler mine, Henry Mountains district

Lucky Strike No. 2 mine, San Rafael district

Sodaroll claim, Green River district

* This name is tentative because zippeite is still poorly defined and we have not proved this to be a polymorph of zippeite. 


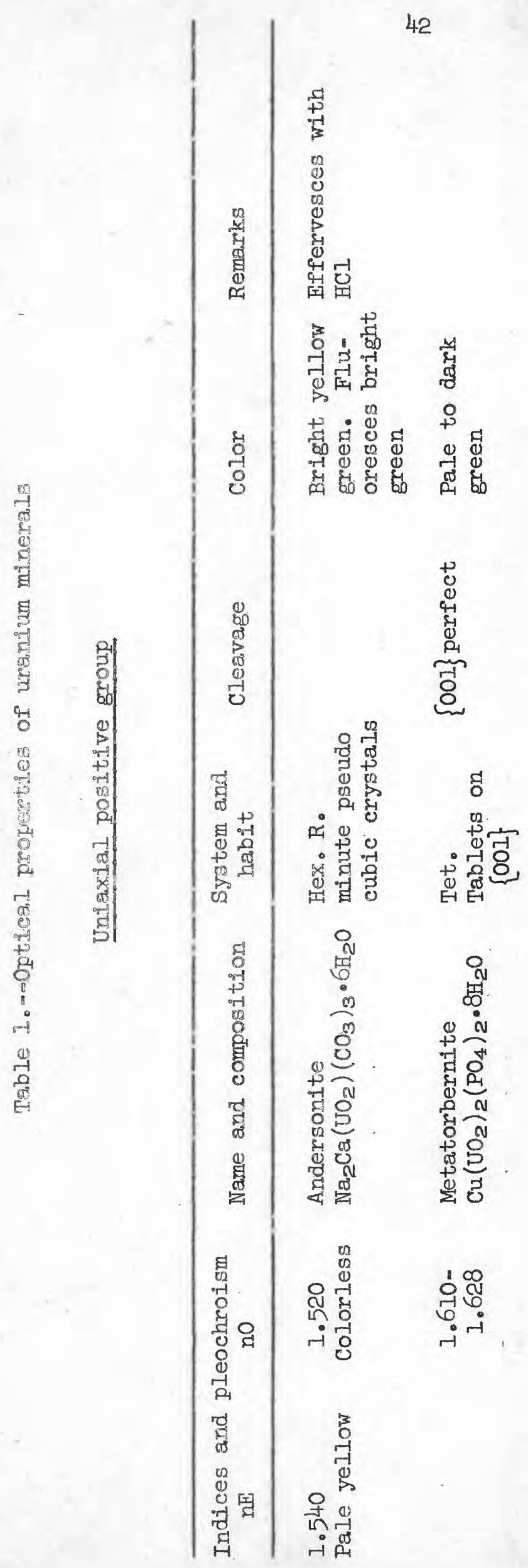




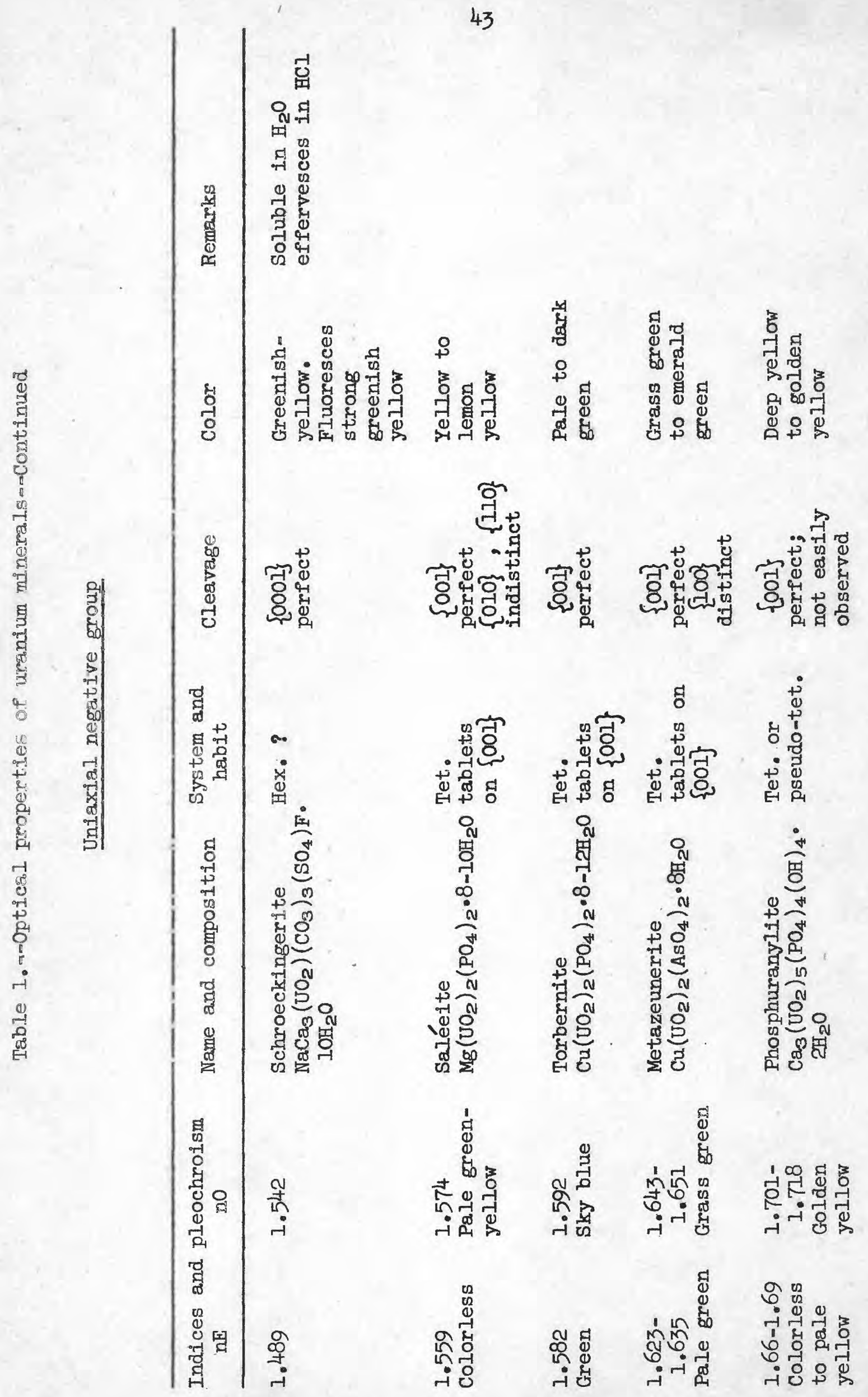




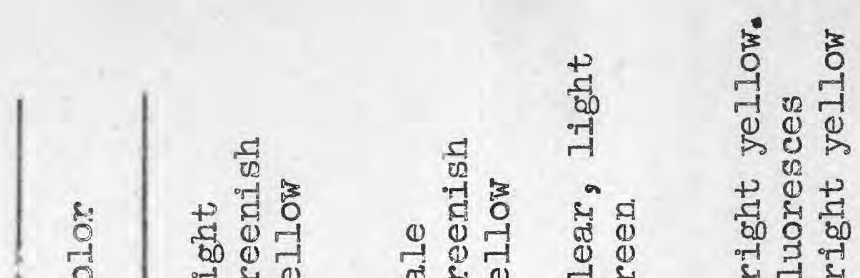

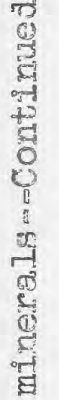

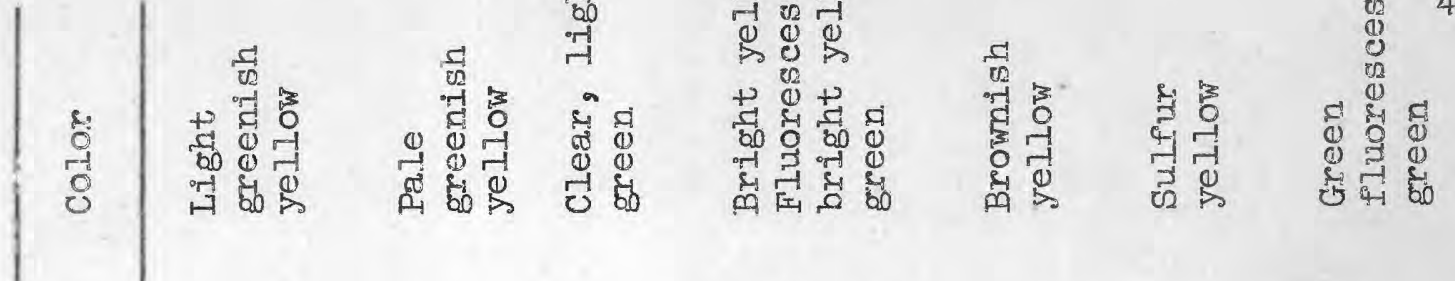

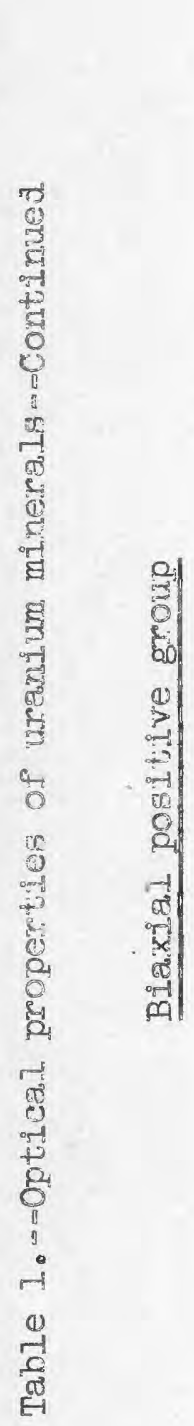

|

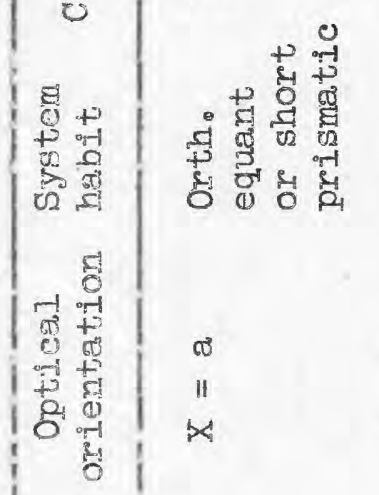

埅

过

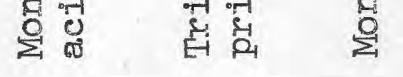

望

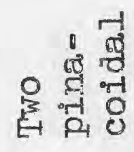

$\circ$ ?

110

$p<$

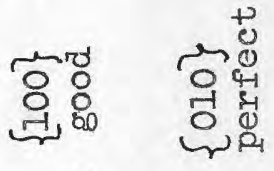

ก.

$N$

II

हैं

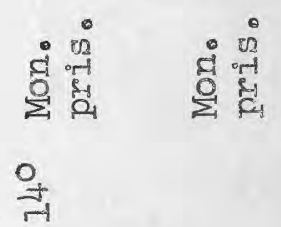

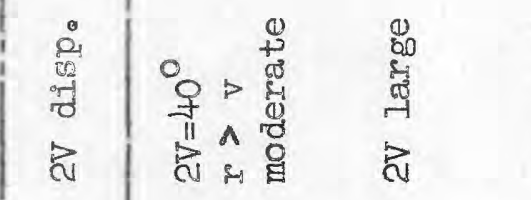

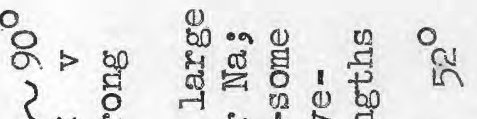

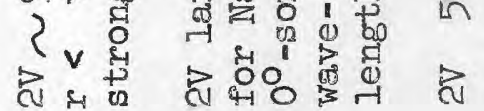

을

0

$<$ ॥

$\times \mathbb{N}$

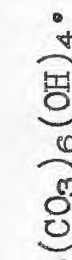

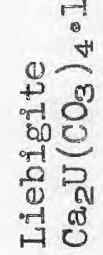

要 零

落

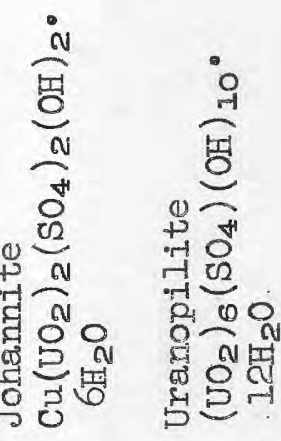

$\underline{2}$

हा

옹

11 势

हो

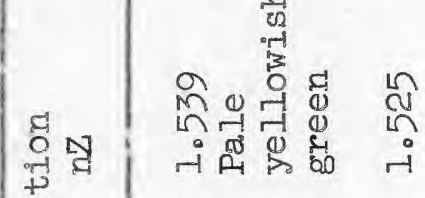

†ू

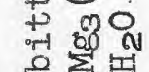

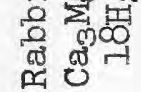

द्व

웅

몰당

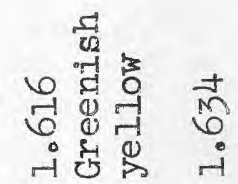

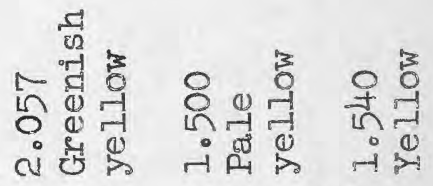

$\mid$

10

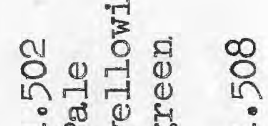

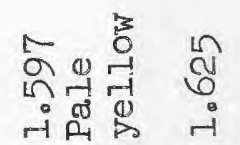

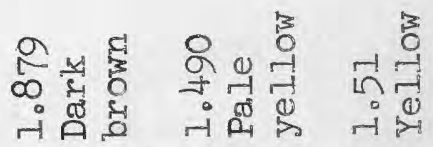

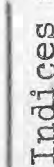

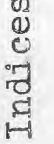

$\rightarrow$ मे के $\rightarrow$

in

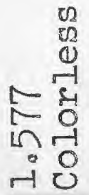

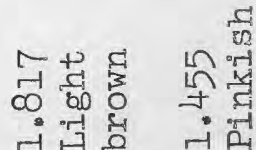

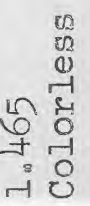




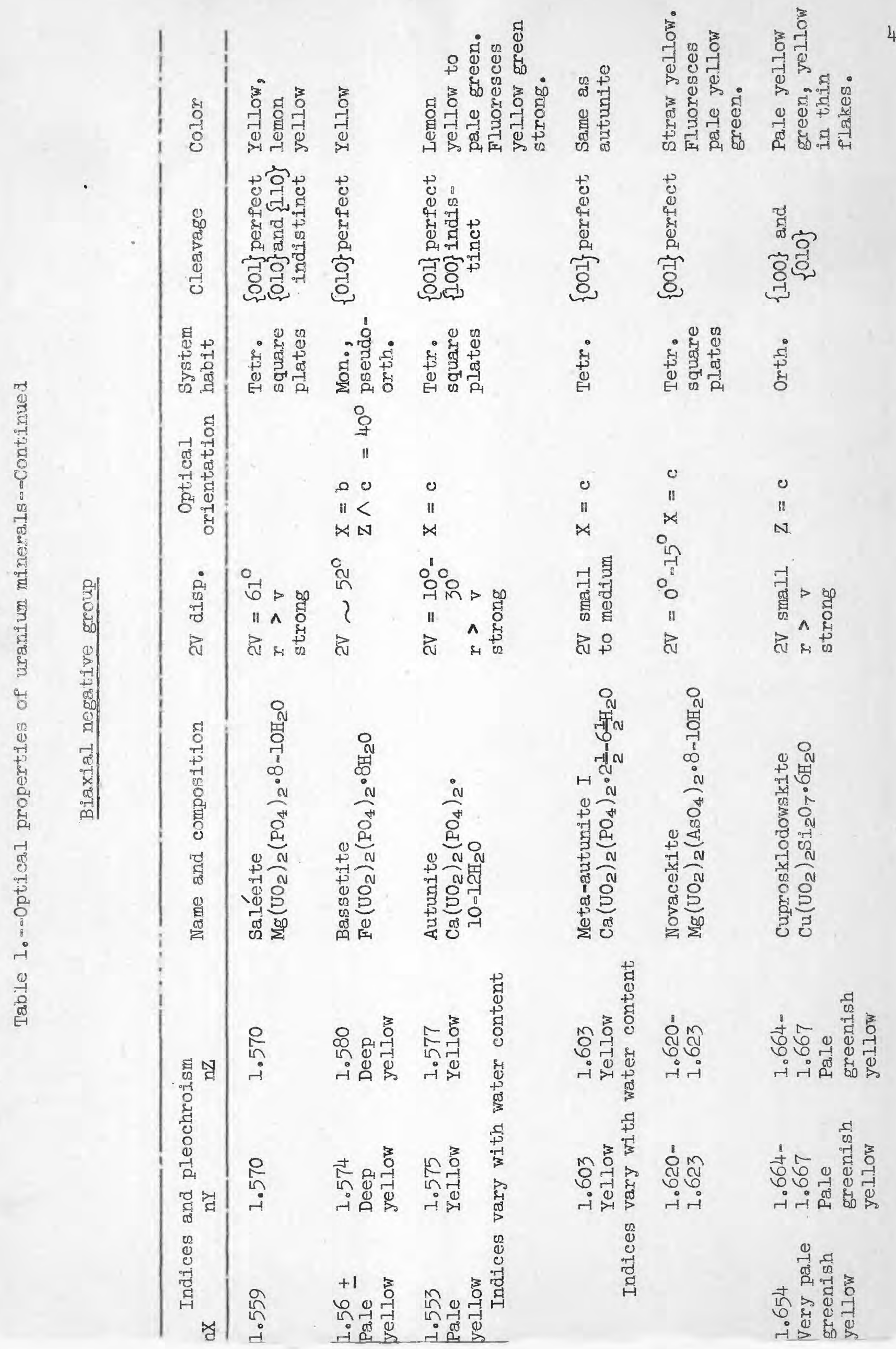




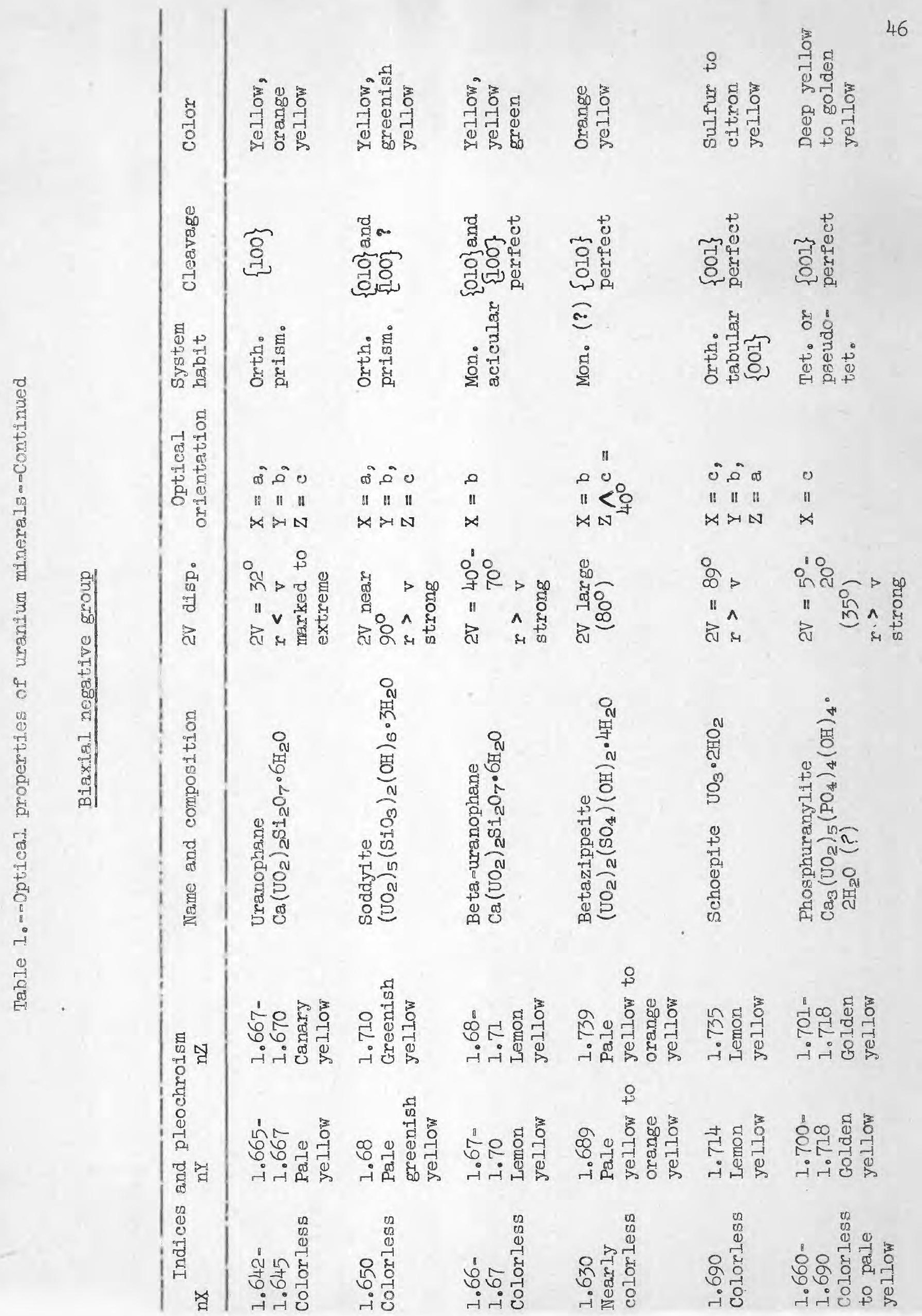


को

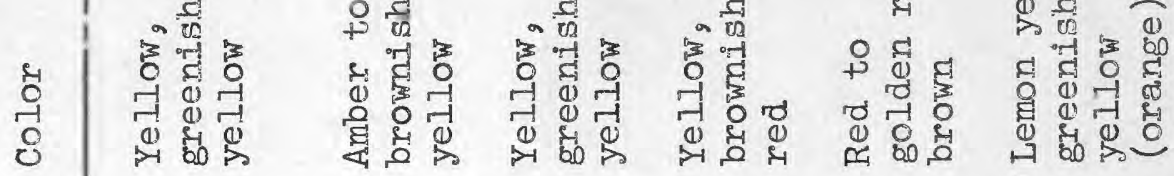

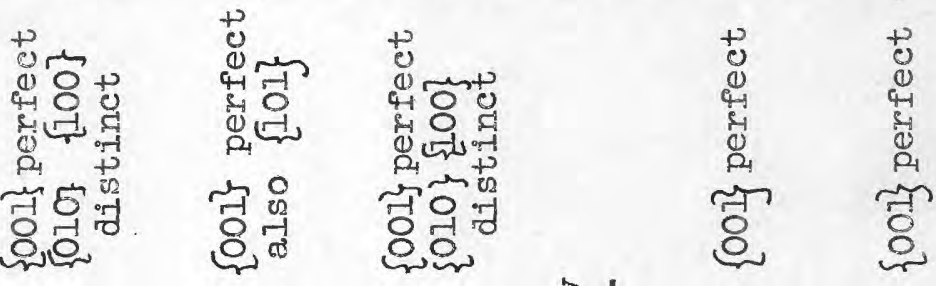

$\frac{\frac{i}{4}}{\dot{s}}$

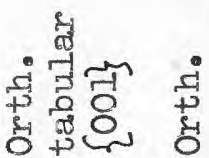

究究

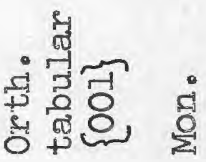

造丝告

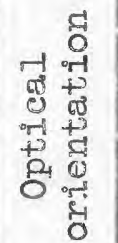

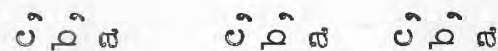

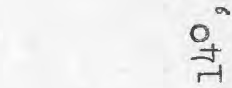

|| || || || || ||

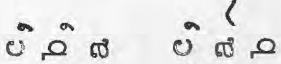

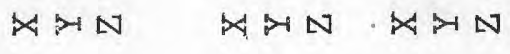

"I II II $<<$ ॥

$X$ M $\triangle A N$

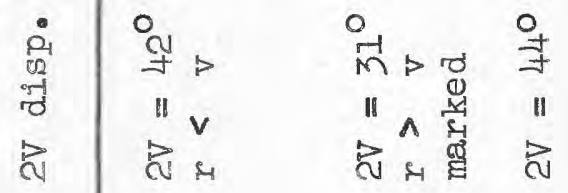

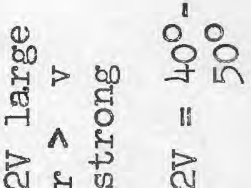

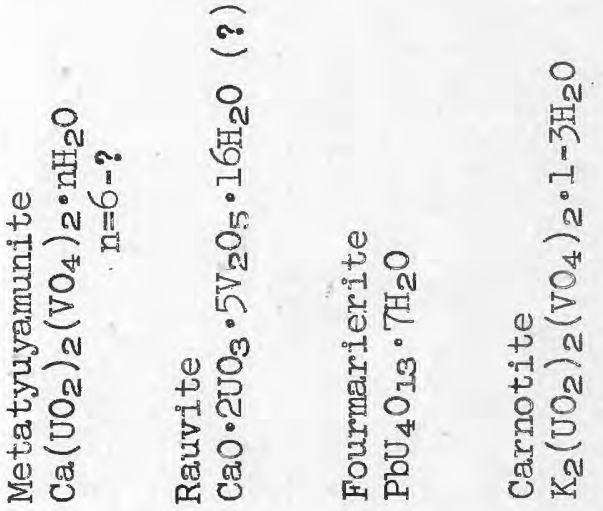

변대

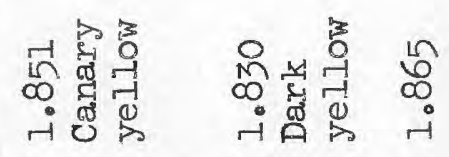

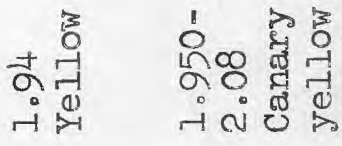

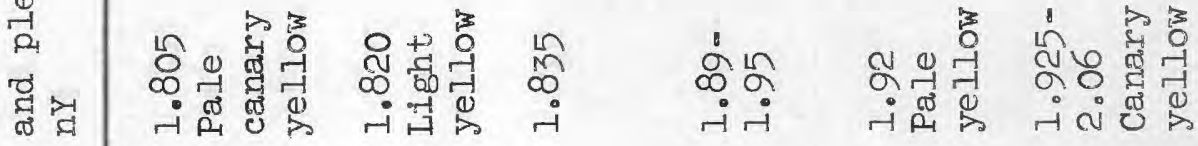

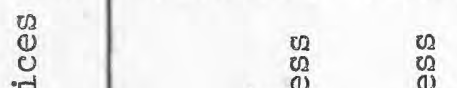

吾

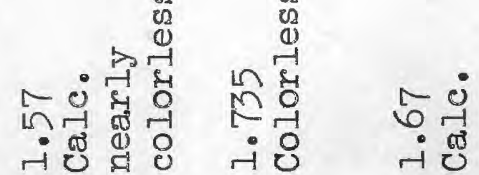

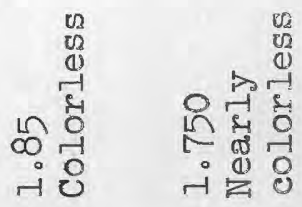




\section{VANADIUM MINERALS}

Description of identified minerals (uranyl vanadates under uranium minerals)

The vanadium minerals described in the following pages (except the uranyl vanadates described under uranium minerals) include all those studied by the writers and thought to be valid species from the Colorado Plateaus, including one mineral, fervanite (Hess and Henderson, 1931) for which no new localities have been found.

Classified according to chemical composition the described minerals are:

Oxides

Vanadates

Silicates
Montroseite $\mathrm{VO}(\mathrm{OH})$ or $(\mathrm{V}, \mathrm{Fe}) \mathrm{O}(\mathrm{OH})$

Navahoite $\mathrm{V}_{2} \mathrm{O}_{5} \cdot 2-3 \mathrm{H}_{2} \mathrm{O}$

Doloresite probably $\mathrm{V}^{+3}$ and $\mathrm{V}^{+4}$, hydrated

Lumsdenite $\mathrm{V}_{2} \mathrm{O}_{3} \cdot \mathrm{V}_{2} \mathrm{O}_{4} \cdot \mathrm{H}_{2} \mathrm{O}$

Calciovolborthite $(\mathrm{Cu}, \mathrm{Ca})_{2}\left(\mathrm{VO}_{4}\right)(\mathrm{OH})$

Volborthite $\mathrm{Cu}_{3}\left(\mathrm{VO}_{4}\right)_{2} \cdot 3 \mathrm{H}_{2} \mathrm{O}$ (?)

(Fervanite $\mathrm{Fe}_{4} \mathrm{~V}_{4} \mathrm{O}_{16} \cdot 5 \mathrm{H}_{2} \mathrm{O}$ ) type specimen only

Steigerite $\mathrm{Al}_{2}\left(\mathrm{VO}_{4}\right)_{2} \cdot 6 \frac{\mathrm{I}_{2} \mathrm{H}_{2} \mathrm{O}}{}$

Rossite $\mathrm{CaV}_{2} \mathrm{O}_{6} \cdot 4 \mathrm{H}_{2} \mathrm{O}$

Metarosite $\mathrm{CaV}_{2} \mathrm{O}_{6} \cdot 2 \mathrm{H}_{2} \mathrm{O}$

Pascoite $\mathrm{Ca}_{2} \mathrm{~V}_{6} \mathrm{O}_{27} \cdot 11 \mathrm{H}_{2} \mathrm{O}$

Hummerite $\mathrm{K}_{2} \mathrm{Mg}_{2} \mathrm{~V}_{10} \mathrm{O}_{28} \cdot 16 \mathrm{H}_{2} \mathrm{O}$

Melanovanadite $2 \mathrm{Ca} .0 \cdot 2 \mathrm{~V}_{2} \mathrm{O}_{4} \cdot 3 \mathrm{~V}_{2} \mathrm{O}_{5}\left[\left(\mathrm{H}_{2} \mathrm{O}\right)\right.$ ?]

Hewettite $\mathrm{CaV}_{6} \mathrm{O}_{16} \cdot 3-9 \mathrm{H}_{2} \mathrm{O}$

Metahewettite $\mathrm{CaV}_{6} \mathrm{O}_{16} \cdot 3-9 \mathrm{H}_{2} \mathrm{O}$ (?)

Sodium analogue of hewettite $\mathrm{Na}_{2} \mathrm{~V}_{6} \mathrm{O}_{16} \cdot 3 \mathrm{H}_{2} \mathrm{O}$

Corvusite $\mathrm{V}_{2} \mathrm{O}_{4} \cdot 6 \mathrm{~V}_{2} \mathrm{O}_{5} \cdot \mathrm{nH}_{2} \mathrm{O}$ (?) (similar to Fernandinite $\left.\mathrm{CaO} \cdot \mathrm{V}_{2} \mathrm{O}_{4} \cdot 5 \mathrm{~V}_{2} \mathrm{O}_{5} \cdot 14 \mathrm{H}_{2} \mathrm{O}\right)$

Roscoelite $(\mathrm{AI}, \mathrm{V})_{2}\left(\mathrm{AlSi}_{3}\right)(\mathrm{K}, \mathrm{Na})_{10}(\mathrm{OH}, \mathrm{F})_{2}$ Vanadium hydromica

Two species originally described from the Colorado Plateaus, vanoxite (Hess, 1925) and pintadoite (Hess and Schaller, 1914) are omitted., The name vanoxite has been used for a variety of vanadium minerals. The composition of vanoxite had been calculated from a rock analysis of sandstone ore from 
Jo Dandy mine, Colo., after deducting quartz, gypsum, tyuyamunite, and limonite. The "type" specimen in the U. S. National Museum came from Wild Steer mine, Colo., and was not analyzed. X-ray powder patterns of this type specimen are similar to those of corvusite and fernandinite. The black crystals observed in thin sections (Hess, 1925, p. 65) probably were montroseite. The description of pintadoite is so incomplete that no more of the mineral can be recognized. No X-ray pattern could be obtained. from the National Museum sample of pintadoite which àpears as a faint green stain on sandstone.

In 1950 when X-ray powder patterns were made for "standards" of all the vanadium minerals, it was found that corvusite (U. S. Nat. Mus. type specimen) and fernandinite (W. T. Schaller's type specimen) give similar patterns. The chief difference between the minerals seems to be the presence of several percent of calcium in fernandinite and little or none in corvusite.

Another vanadium mineral that may occur on the Plateaus although it has not been identified yet is sincosite $\mathrm{CaV}_{2} \mathrm{O}_{2}\left(\mathrm{PO}_{4}\right)_{2} \cdot 5 \mathrm{H}_{2} \mathrm{O}$. 
CORVUSITE

$$
\mathrm{V}_{2} \mathrm{O}_{4} \cdot 6 \mathrm{~V}_{2} \mathrm{O}_{5} \cdot \mathrm{nH}_{2} \mathrm{O}
$$

(Resembles fernandinite $\mathrm{CaO} \cdot \mathrm{V}_{2} \mathrm{O}_{4} \cdot 5 \mathrm{~V}_{2} \mathrm{O}_{5} \cdot 14 \mathrm{H}_{2} \mathrm{O}$ )

Crystal system:

Habit: Massive. Very finely crystalline. Slickensided surfaces appear fibrous.

Physical properties:

Color: blue black to greenish black. Weathers brown Fluorescence: none

Luster: variable

Cleavage: fracture conchoidal

Hardness: $2 \frac{1}{2}-3$

Specific gravity: 2.82 (?)

Strongest lines of X-ray powder pattern: VS 12.1, M 3.47, W 1.83, VW 1.95

Optical properties: Opaque except on thin edges; biaxial, 2 indices above 1.90, high birefringence.

Analysis: Qualitative spectrographic analysis of $X-r a y$ spindle of type material.

$\begin{array}{ll}\text { Major } & \mathrm{V} \\ \text { Minor } & \text { Fe } \\ \text { Low minor } & \text { Si AI }\end{array}$

Occurrence and associated minerals: Impregnating sandstone and siltstone. Masses of relatively pure material are commonly slickensided. May be an alteration product of low valence vanadium oxides. Associated with carnotite, tyuyamunite, rauvite, and hewettite.

Identification: In hand specimen, black, commonly with blue-black iridescence, and greenish streak. $\mathrm{X}$-ray powder pattern resembles that of fernandinite. (The corvusitefernandinite problem is being investigated, June 1953).

Localities:

Abundant in mines in Thompsons, Gateway, Uravan, Paradox, Bull Canyon, and Slick Rock districts, in the Temple Mountain part of the San Rafael district, and at Monument No. 2 mine in Monument Valley district. 
Crystal system:

Habit: Massive, fibrous, radial aggregates, in veinlets

Physical properties:

Color: nearly black with bronze tint; bronze in polished section Fluorescence: none

Luster: adamantine

Cleavage: perfect in one direction

Hardness:

Specific gravity: 3.25

Strongest lines of X-ray powder pattern: S 4.72, s 2.47, M 3.83, M 3.17

Optical properties: opaque

Analysis:

Occurrence and associated minerals: Occurs with coffinite and lumsdenite* at Ia Sal No. 2 mine and with clausthalite and pitchblende at Corvusite mine. (Doloresite was first found by L. R. Stieff and T. W. Stern in August 1951 at Ia Sal No. 2 mine; named from Dolores Rives; report in preparation.)

Identiflcation: $X$-ray powder pattern, not as black as montroseite, heavier than melanovanadite.

Localities:

La Sal No. 2 mine, Gateway district

Utex mine, Monticello district

Corvusite mine, Gateway district

*Another new mineral, lumsdenite, was found with doloresite at La Sal No. 2 mine. It is known only from the $X$-ray powder pattern and single crystal X-ray photographs taken from a few microscopic crystals. The intensities are consistent with an atomic arrangement that contains elements of the montroseite structure and the rutile-type structure of artificial $\mathrm{VO}_{2}$. It is orthorhombic and its formula is probably $\mathrm{V}_{2} \mathrm{O}_{3} \cdot \mathrm{V}_{2} \mathrm{O}_{4} \cdot \mathrm{H}_{2} \mathrm{O}$ (personal communication, H. T. Evans, U.S.G.S.). Named. from Iumsden group of mines, in which La Sal No. 2 mine is located, at the head of Lumsden Canyon, Colo. 
Crystal system: Probably monoclinic

Habit: Parallel fibrous aggregates

Physical properties:

Color: golden brown

Fluorescence:

Luster: brilliant

cleavage:

Hardness :

Speciflc gravity:

Strongest lines of X-ray porder pattern (taken with $\mathrm{Fe} \mathrm{Ka}$ radiation): $\mathrm{S} 8.83, \mathrm{~S} 6.44, \mathrm{M} 2.92$

Optical properties:

$\underline{\mathbf{n}}$

$\begin{array}{lr}X & 2.186 \pm 0.005 \\ Y & 2.222 \pm 0.005 \\ Z & 2.224 \pm 0.005 \\ 2 V \text { very small }\end{array}$

Biaxial negative.

2V very small

Analysis: Chemical analysis of type material from Gypsum Valley.

E. P. Henderson, analyst.

$\begin{array}{rrrr}\mathrm{Fe}_{2} \mathrm{O}_{3} & \mathrm{~V}_{2} \mathrm{O}_{5} & \mathrm{H}_{2} \mathrm{O}(-) & \text { Total } \\ 41.89 & 46.10 & \mathrm{I2.01} & 100.00\end{array}$

Recalculated after deducting 9.40 percent insol. and 7.34 percent Bypsum.

Occurrence and associated minerals:

Coatings and fracture fllings; with sypsum, metahewettite, carnotite, and various black vanadium minerals.

Identification: Iighter brown color and higher index of refraction than fibrous hewettite.

Localities:

Polar Mesa, Gateway district, and in Gysum Valley district.

*A1I data except X-ray porder pattern from Dana, 7 th ed., vol. 2 , p. 1049, No new occurrences of this wineral found by iriters. 
HEWETIITE

$\mathrm{CaV}_{6} \mathrm{O}_{16} \cdot 3-9 \mathrm{H}_{2} \mathrm{O}$

Crystal system: Monoclinic*

Habit: As nodular aggregates and coatings of fibers or microscopic needles; elongated $\{010\}$

Physical properties:

Color: deep red; less vivid on exposure in dry atmosphere

Fluorescence: none

Luster: silky, adamantine

cleavage:

Hardness : soft

Specific gravity: 2.55

Strongest lines of X-ray powder pattern: VS 8.2, M 3.06, M 2.29, M 2.20

Optical properties:

$\begin{array}{lcl}\text { Orientation } & \underline{n} & \text { Pleochroism } \\ X & 1.77 & \text { Light orange yellow } \\ Y & 2.18 & \text { Light orange yellow } \\ \mathrm{Z}=\mathrm{b} & 2.35-2.4 & \text { Dark red } \\ \text { ZV medium } & & \end{array}$

Biaxial negative Indices probably vary according to water content.

Ana.lysis: Chemical analysis of material from Jo Dandy group. Analyst: A. M. Sherwood

$\begin{array}{lrlllllll}\mathrm{SiO}_{2} & \mathrm{Al}_{2} \mathrm{O}_{3} & \mathrm{CaO} & \mathrm{MgO} & \mathrm{V}_{2} \mathrm{O}_{4} & \mathrm{~V}_{2} \mathrm{O}_{5} & \mathrm{SO}_{3} & \mathrm{H}_{2} \mathrm{O} & \text { Total } \\ 0.46 & 0.13 & 6.38 & 1.61 & 8.07 & 73.15 & 0.01 & 10.12 & 99.93\end{array}$

Occurrence and associated minerals:

As coatings and fracture fillings; alteration product of less oxidized vanadium minerals-montroseite, corvusite. Associated with vanadium clay, rauvite, steigerite, navahoite, carnotite, tyuyamunite, etc.

Identification:

Color except from sodium analogue of hewettite. The nature of the difference, if any, between hewettite and metahewettite is not fully understood. The structures of these minerals are being investigated by $\mathrm{W} . \mathrm{H}$. Barnes, National Research Council, Canada.

Localities: Jo Dandy mine, Bull Canyon district; Opera Box mine, Bull Canyon district; Matchless mine, Gateway district; Monument No. 2 mine, Monument Valley district. 
Crystal system: Monoclinic*

Habit: As pulverulent masses composed of microscopic tablets or laths, and as parallel or radially fibrous to bladed aggregates or coatings; elongated $\{010\}$ 。

Physical properties:

Color: deep red; less vivid on exposure in dry atmosphere Fluorescence: none

Luster: dull to somewhat silky

Cleavage:

Hardness: soft

Specific gravity: 2.51-2.94, varies with water content

Strongest lines of X-ray powder pattern: S 8.1, M 3.08, W 1.80

Optical properties:

\begin{tabular}{|c|c|c|c|}
\hline Orientation & $\underline{n}(\mathrm{Li})$ & Pleochroism & \\
\hline $\begin{array}{l}X \\
Y \\
Z=b \\
2 V 52^{\circ} \text { calc }\end{array}$ & $\begin{array}{l}1.70 \\
2.10 \\
2.23\end{array}$ & $\begin{array}{l}\text { Light orange } \\
\text { Deep red } \\
\text { Deeper red }\end{array}$ & $\begin{array}{l}\text { yellow } \\
\text { Biaxial negative } \\
\text { Indices probably vary } \\
\text { according to water } \\
\text { content. }\end{array}$ \\
\hline
\end{tabular}

Analysis: Qualitative spectrographic analysis of type material

$\begin{array}{ll}\text { Major } & \mathrm{V} \\ \text { Minor } & \mathrm{Si} \mathrm{Ca} \mathrm{Fe} \mathrm{Al} \\ \text { Trace } & \mathrm{K} \text { Mg Na } \mathrm{Nb} \mathrm{Ba} \mathrm{Pb}\end{array}$

Occurrence and associated minerals: In highly oxidized ore; same as for hewettite.

Identification: Color, except from hewettite, and sodium analogue of hewettite. U. S. Nat. Mus. type material gives same X-ray pattern as hewettite.

Locality:

Yellow Cat group and Cactus Rat group, Thompsons district.

*Barnes, W. H., and Qurashi, M. M., 1952, p. 411. 
HUMMERITE*

$\mathrm{K}_{2} \mathrm{Mg}_{2} \mathrm{~V}_{10} \mathrm{O}_{28} \cdot 16 \mathrm{H}_{2} \mathrm{O}$

Crystal system: Triclinic

Habit: Finely crystalline aggregates, or massive. Crystals formed by evaporation of water solution are elongated parallel to $\{001\}$ or tabular paralle 1 to $\{100\}$.

Physical properties:

Color: bright orange. Streak yellow

Fluorescence: none

Luster: subadamantine on fresh surface, dulls on exposure.

Cleavage: $\{010\}$ and $\{001\}$ distinct. Brittle.

Hardness: about 2

Specific gravity: 2.55

Strongest lines of X-ray powder pattern: VS $8.3, \mathrm{M} 9.7, \mathrm{M} 2.76, \mathrm{~W} 7.5$

Optical properties:

Orientation

$\underline{\mathrm{n}}$

$\mathrm{X} \quad 1.771+0.003$

$I \quad 1.812 \mp 0.003$

$\mathrm{Z} \wedge c=32^{\circ} \quad 1.833 \mp 0.003$

Biaxial negative

$2 V=70^{\circ}$; dispersion strong $r>v$

Analysis: Chemical analysis of recrystallized material from North Star mine

$\begin{array}{rrrrrr}\mathrm{V}_{2} \mathrm{O}_{5} & \mathrm{~V}_{2} \mathrm{O}_{4} & \mathrm{MgO} & \mathrm{K}_{2} \mathrm{O} & \text { Total } \mathrm{H}_{2} \mathrm{O} & \text { Tota.1 } \\ 64.33 & 1.36 & 5.44 & 6.96 & 21.88 & 99.97\end{array}$

Qecurrence and associated minerals: As vein fillings with columnar structure perpendicular to the vein wall (similar to occurrence of gypsum in seams). Also as granular crusts coating or cementing sandstone, in highly oxidized ore zone.

Associated with hewettite and vanadium clay.

Identification: $X$-ray powder pattern or spectrographic analysis necessary to distinguish from pascoite.

Localities: Jo Dandy group, Bull Canyon district; North Star mine, Uravan district, Mesa No. 1 mine, Shiprock district and Whitney mine, Uravan district.

* First collected by Stieff, Stern, and Girhard in 1949 from the Humer workings of Jo Dandy group of mines and studied by Weeks, Cisney, and Sherwood (1950). Named from the first locality. 
MELANOVANADITE

\author{
$2 \mathrm{CaO} \cdot 2 \mathrm{~V}_{2} \mathrm{O}_{4} \cdot 3 \mathrm{~V}_{2} \mathrm{O}_{5}$ \\ probably contains $\mathrm{H}_{2} \mathrm{O}^{*}$
}

Crystal system: Triclinic*

Habit: Velvety, divergent bunches of crystals elongated [001]

the prism faces usually rounded or striatea.

Physical properties:

Color: black; streak dark reddish brown

Fluorescence: none

Luster: almost submetallic

Cleavage: $\{010\}$ perfect. Brittle

Hardness: $2 \frac{1}{2}$

Specific gravity: commonly less than 3.0

Strongest lines of X-ray powder pattern: VS $8.5, \mathrm{~S} 4.21, \mathrm{M} 2.99$

Optical properties:

Orientation $\quad \underline{\mathrm{n}} \quad \underline{\text { Pleochroism }}$

\begin{tabular}{|c|c|c|c|}
\hline $\begin{array}{l}X \\
Y \\
Z\end{array} \wedge c \quad 15^{\circ}$ & $\begin{array}{l}1.73 \\
1.96 \\
1.98\end{array}$ & $\begin{array}{l}\text { Iight reddish brown } \\
\text { Deep reddish brown } \\
\text { Dark reddish brown }\end{array}$ & Biaxial negative \\
\hline
\end{tabular}

Analysis: Qualitative spectrographic analysis of material from Mesa No. I mine, Ariz.

$\begin{array}{ll}\text { Major } & \mathrm{V} \\ \text { Minor } & \mathrm{Ca} \mathrm{Fe} \\ \text { Trace } & \mathrm{Si} \mathrm{Al} \mathrm{Na} \mathrm{Mg}\end{array}$

Dccurrence and associated minerals:

At Mesa No. I mine, coarsely crystalline aggregates in clay with marcasite. At Mesa No. 5 mine, impregnating sandstone. At Juniper mine rosettes on fracture in sandstone. In oxidized ore associated with tyuyamunite, pascoite, humerite, and rossite. The first occurrence of melanovanadite in the U. S. was at Mesa No. I mine found by A. Rosenzweig of A.E.C. in 1951.

Identification: Distinguished from montroseite and doloresite by pleochroism and specific gravity.

Localities: Mesa No. 1 mine, Shiprock district, Mesa No. 5 mine, Shiprock district, and Juniper mine, Thompsons district.

*Barnes, W. H., and Qurashi, M. M., 1952, p. 417. 
MONTROSEITE*

$$
\mathrm{VO}(\mathrm{OH}) \text { or }(\mathrm{V}, \mathrm{Fe}) \mathrm{O}(\mathrm{OH})
$$

Crystal system: Orthorhombic, dipyramidal

Habit: Microscopic bladed and prismatic crystals. Also in compact crystalline aggregates.

Physical properties:

Color: black; streak black

Fluorescence: none

Luster: submetallic

Cleavage: perfect parallel $\{100\}$. Brittle

Hardness: soft

Specific gravity: 4.0 meas. 4.1 calc.

Strongest lines of X-ray powder pattern: $S 4.29, \mathrm{M} 2.65$, W 3.39

Optical properties: Opaque (even on thin edges).

Analysis: Partial chemical analysis of $120 \mathrm{mg}$ of material from Bitter Creek mine.

$\begin{array}{lllll}\mathrm{FeO} & \mathrm{V}_{2} \mathrm{O}_{3} & \mathrm{~V}_{2} \mathrm{O}_{4} & \mathrm{H}_{2} \mathrm{O} & \text { Total } \\ 8.8 & 10.5 & 72.3 & 5.0 & 96.6\end{array}$

Occurrence and associated minerals: Occurs in unoxidized, black, uraniumvanadium ore, impregnating sandstone or as relatively pure masses in sandstone, associated with pyrite, barite, and coffinite (?). In oxidized zone alters to corvusite and hewettite.

Identification: X-ray powder pattern. Distinguished from doloresite by blacker color and from melanovanadite by greater density and opacity.

Localities: Bitter Creek mine, Uravan district; Whitney mine, Uravan district; Matchless mine, Gateway district; Juniper mine, Thompsons district; and Rex No. 2 mine, Temple Mountain portion of San Rafael district.

*First collected by Stieff, Stern, and Girhard in 1949 from Bitter Creek mine. Preliminary study by Weeks, Cisney, and Sherwood (1950). Crystal structure study by H. T. Evans in 1952 showed the correct formula to be as given above. Named from Montrose County, Colo., where Bittex Creek mine is located. 
MAVAHOITE

$$
\mathrm{V}_{2} \mathrm{O}_{5} \cdot 2-3 \mathrm{H}_{2} \mathrm{O}
$$

Crystal system: Monoclinic (?)

Habit: Fibrous, silky

Physical properties:

Color: dark brown; brown streak

Fluorescence: none

Iuster: silky

Cleavage: .

Hardness: soft

Specific gravity: 2,56 measured

Strongest lines of X-ray powder pattern: VS 12.1, M 10.7, M 2.91

Optical properties:

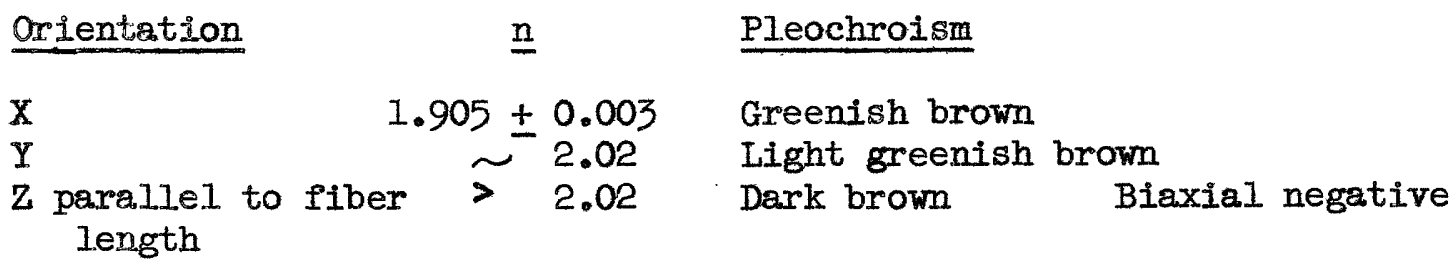

Analysis: Of sample from Arizona (AW-119-52) A. M. Sherwood, analyst.

$\begin{array}{rrrrrrr}\mathrm{V}_{2} \mathrm{O}_{5} & \mathrm{~V}_{2} \mathrm{O}_{4} & \mathrm{Fe}_{2} \mathrm{O}_{3} & \mathrm{CaO} & \mathrm{SiO}_{2} & \mathrm{H}_{2} \mathrm{O} & \text { Total } \\ 71.68 & 3.08 & 3.58 & 0.22 & 1.20 & 20.3 & 100.06\end{array}$

Occurrence and associated minerals: Fibrous coating in crescent shape above and below pebbles in conglomerate; cross fibers $1 / 16$ to $1 / 8$ inch long filling fractures in sandstone or siltstone; with rauvite, corvusite, and steigerite.

Identification: Darker brown than hewettite. $\mathrm{X}$-ray powder pattern.

Locality:

Monument No. 2 mine, Arizona, Monument Valley district. Mineral named for Navaho Indian Reservation on which the Monument No. 2 mine is located. First sample collected by A. Rosenzweig, A.E.C., in 1951. Material for chemical analysis and X-ray study collected by A. D. Weeks 1951 and 1952. 
PASCOITE

$$
\mathrm{Ca}_{2} \mathrm{~V}_{6} \mathrm{O}_{17} \cdot 11 \mathrm{H}_{2} \mathrm{O}
$$

Crystal system: Triclinic

Habit: As granular crusts, rarely showing minute lath-like crystals with oblique terminations.

Physical properties:

Color: dark red orange to yellow orange

Fluorescence: none

Luster: vitreous to subadamantine

Cleavage: $\{010\}$ distinct. Fracture conchoidal

Hardness: $\sim 2 \frac{1}{2}$

Specific gravity: 1.87

Strongest lines of X-ray powder pattern: VS $8.7, \mathrm{M} \mathrm{7.4}$, W 9.4, W 4.69

Optical properties:

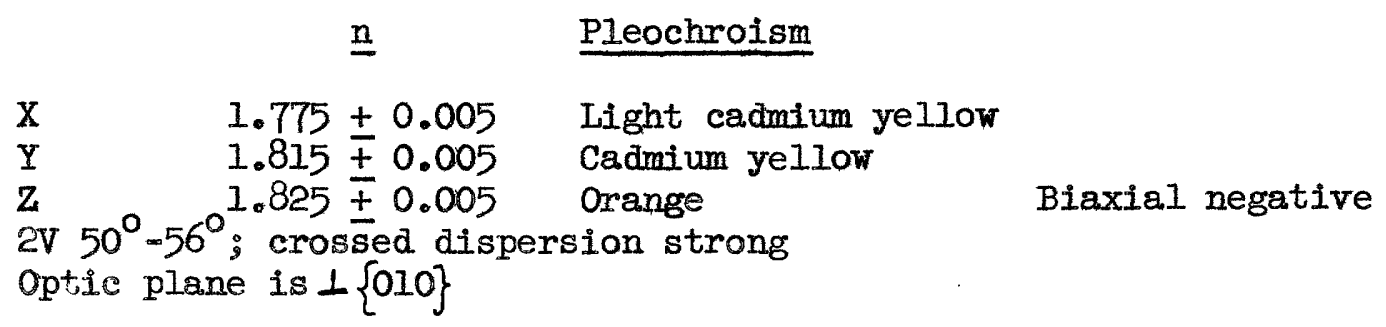

Analysis: Qualitative spectrographic analysis of mineral from Mesa No. I mine, Ariz.

$\begin{array}{lllll}\text { Major } & \text { V } & \text { Ca } & & \\ \text { Minor } & \text { Fe } & & & \\ \text { Trace } & \text { Na } & \text { Al } & \text { Mg } & \text { SI }\end{array}$

Occurrence and associated minerals: Coating mine walls and open fractures: in oxidized zone; coating montroseite, melanovanadite, and other vanadium minerals.

Identification: X-ray powder pattern or spectrographic analysis necessary to distinguish from humerite. Orange color and solubility in water distinguish it from all others.

Localities:

Mesa No. 1 mine, Shiprock district; Bitter Creek mine, Uravan district; Mill No. I mine, Uravan district; and Corvusite mine, Gateway district. 
ROSCOELITE

and VANADIUM HYDROMICA*

$$
(\mathrm{Al}, \mathrm{V})_{2}\left(\mathrm{AlSi}_{3}\right)(\mathrm{K}, \mathrm{Na}) \mathrm{O}_{10}(\mathrm{OH}, \mathrm{F})_{2}
$$

Crystal system: Monoclinic

Habit: Massive

Physical properties:

Color: green, gray, tan, brown

Fluorescence: none

Luster: pearly

Cleavage: basal

Hardness: soft

Specific gravity:

Strongest lines of X-ray powder pattern: S 10.0, S 3.34, M 4.50, M 2.59

Optical properties:

Transparent if finely divided. Birefringent.

Analysis: Chemical (Fischer et al., 1947, p. 124). Roscoelite from Placerville, Colo.

$\begin{array}{llllllllll}\mathrm{SiO}_{2} & \mathrm{Al}_{2} \mathrm{O}_{3} & \mathrm{FeO} & \mathrm{V}_{2} \mathrm{O}_{3} & \mathrm{MgO} & \mathrm{CaO} & \mathrm{K}_{2} \mathrm{O} & \mathrm{Na}_{2} \mathrm{O} & \mathrm{H}_{2} \mathrm{O} & \text { Rem. Total }\end{array}$

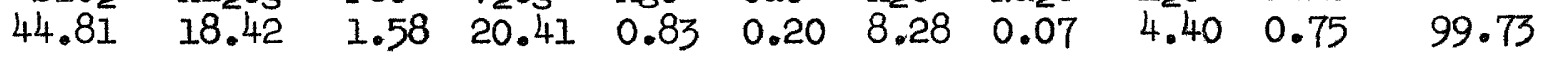

(Analyst: V. North)

Less $0=F \quad 0.06$

$\overline{99.67}$

Occurrence and associated minerals: Impregnating sandstone and replacing clay pellets and stringers. Associated with corvusite, hewettite, carnotite, and tyuyamunite.

Identification: Although the X-ray powder pattern distinguishes this pair from other vanadium minerals, commonly it does not distinguish between these two minerals. Also, some "vanadium clay ore" may consist of hydromica with included vanadium oxides. (M. D. Foster, U.S.G.S.)

Localities:

Districts: Gateway, Placerville, Thompsons, Uravan, Paradox, Bull Canyon, Gypsum Valley, and Slick Rock.

*Hydromica contains less potassium and is more hydrated than roscoelite. 
ROSSITE

$$
\mathrm{CaV}_{2} \mathrm{O}_{6} \cdot 4 \mathrm{H}_{2} \mathrm{O}
$$

Crystal system: Triclinic

Habit: Glassy lumps surrounded by flaky alteration rims of metarossite.

Physical properties:

Color: yellow

Fluorescence: none

Luster: vitreous to somewhat pearly

Cleavage: $\{010\}$ good. Brittle.

Hardness: $2-3$

Specific gravity: 2.45

strongest lines of X-ray powder pattern: $\mathrm{S} 7.3, \mathrm{~s} 6.66, \mathrm{~s} 3.87$

Optical properties:

Orientation $\underline{\underline{n}} \quad \underline{\text { Color }}$

$\begin{array}{lll}\mathrm{X} & & \\ \mathrm{Y} \wedge \mathrm{b} \sim 45^{\circ} & 1.710 & \\ \mathrm{Z} \sim \mathrm{c} & 1.770 & \text { Biaxial negative (?) } \\ 2 V & 1.840 & \text { Yellow }\end{array}$

Analysis: No new analysis. See Dana VII

Occurrence and associated minerals: Secondary coatings and veinlets, in oxidized zone, with metarossite. USMM samples of rossite described

in 1927 have all dehydrated to metarosite. (1950).

Identification: Optical properties, color. Readily soluble in hot water. Localities:

Originally described by Foshag and Hess from an occurrence at Bull Pen Canyon, Slick Rock district, Mesa No. I mine, Shiprock district. 


$$
\mathrm{CaV}_{2} \mathrm{O}_{6} \cdot 2 \mathrm{H}_{2} \mathrm{O}
$$

Crystal system:

Habit: Soft and friable, platy to flaky masses in veinlets.

Physical properties:

Color: very light yellow, pale greenish yellow

Fluorescence: none

Iuster: more pearly than rossite

Cleavage:

Hardness: soft

Specific gravity:

Strongest lines of X-ray powder pattern: S 5.9, S 5.1, M 3.05

Optical properties:

$\underline{n}$

$X$

1.840

$\mathrm{Y}>1.85$

$\mathrm{Z}>1.85$

Biaxial positive

2V large; dispersion strong

Analysiø: Qualitative spectrographic analysis of material from Buckhorn: claim.

$$
\begin{array}{ll}
\text { Major } & \mathrm{V} \\
\text { Minor } & \mathrm{Ca} \\
\text { Trace } & \mathrm{Al} \text { Si No } \mathrm{Fe} \mathrm{Mg}
\end{array}
$$

occurrence and associated minerals: Same as rosite

Identification: Color, optical properties. Readily soluble in hot water. Localities :

Buckhorn claim, Slick Rock district

Spring Creek, Brushy Basin, Monticello district 
Crystal system: Monoclinic

Habit: Bladed or acicular: botryoidal

Physical properties:

Color: deep red; brownish red on exposure Fluorescence: none

Luster: adamantine, dulls on exposure Cleavage:

Hardness :

Specific gravity:

Strongest lines of X-ray powder pattern: vs 7.97 , s 3.13, s 2.27

Optical properties:

$\begin{array}{lrll}\text { Orientation } & \underline{n} & \text { Pleochroism } & \\ \mathrm{X} & \text { approx. } 1.8 & \text { Yellow } & \\ \mathrm{Y} & >2.0 & \text { Orange yellow } & \text { Biaxial negative } \\ \mathrm{Z}=\mathrm{b} & >2.0 & \text { Orange red } & \text { 2V medium }\end{array}$

Analysis: Chemical analysis of material from Cactus Rat incline. Analyst: A. M. Sherwood

$\begin{array}{llllllll}\mathrm{V}_{2} \mathrm{O}_{4} & \mathrm{~V}_{2} \mathrm{O}_{5} & \mathrm{Na}_{2} \mathrm{O} & \mathrm{K}_{2} \mathrm{O} & \mathrm{CaO} & \mathrm{Acid} \text { insol. } \mathrm{H}_{2} \mathrm{O}- & \mathrm{H}_{2} \mathrm{O}+ & \text { Total }\end{array}$

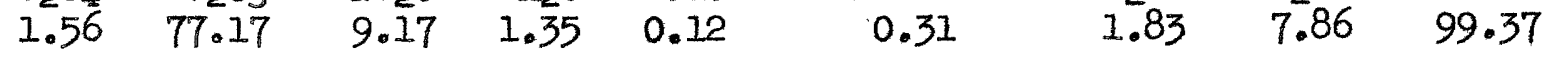

Decurrence and associated minerals: Coating a fracture in the roof of the Cactus Rat incline, with steigerite.

Identification: By color, except from hewettite. By X-ray or spectroscopic analysis from hewettite.

Localities: The first sample was collected by Benjamin Webber from the Thompsons district, during World War II. The second sample was collected by J. Stone in July 1952, from Cactus Rat incline, Thompsons district. 
Crystal system:

Habit: As canary yellow pulverulent coatings that are variously composed of cryptocrystaline fibrous material resembling chalcedony, gumlike masses, and occasionally flat plates.

Physical properties:

Color: canary yellow

Fluorescence: none

Luster: waxy in compact aggregates

Cleavage:

Hardness:

Specific gravity:

Strongest Iines of X-ray powder pattern: S 10.5, S 12.4, W 5.6

Optical properties:

Mesn index $1.710 \pm 0.005$

Analyais: Qualitative spectrographic analysis of material from Cactus Rat incline.

$$
\begin{array}{ll}
\text { Major } & \mathrm{V} \\
\text { Minor } & \mathrm{Al} \mathrm{Ca} \\
\text { Trace } & \mathrm{U} \text { Na Fe Si }
\end{array}
$$

oceurrence and associated minerals: At Cactus Rat, coatings on highly weatiked sandstone, with sodium analogue of hewettite.

Identification: Color and lack of radioactivity.

Loca.IIty: Original locality - north wall of Gypsum Valley, Gypsum Valley distriots.

Cactus Rat incline, Thompsons district; Monument No. 2 mine, Monument Valley district. 
Crystal system: Monoclinic (?)

Habit: As scaly, spongy, or fibrous crusts and as rosette-like aggregates; also reticulated. Some as scales with a triangular or hexagonal outline.

Physical properties:

Color: dark olive green to green and yellowish green

Fluorescence: none

Luster: vitreous to pearly on the cleavage

Cleavage: perfect in one direction

Hardness: $3 \frac{1}{2}$

Specific gravity: $3.5-3.8$

Strongest lines of X-ray powder pattern: S 7.2, M 2.88, M 2.56, M 2.39, M 1.51

Optical properties:

$\begin{array}{llll} & \underline{n} & \text { Color } \\ \mathrm{X} & 2.01 & \text { Green to } & \\ \mathrm{Y} & 2.05 & \text { greenish yellow Biaxial positive red } \\ \mathrm{Z} & 2.10 & \text { in transmitted light. } \\ \mathrm{IV} 68^{\circ} \mathrm{Li}, 83^{\circ} \mathrm{Na} ; & \mathrm{r}>\mathrm{V} \text { inclined }\end{array}$

Analysis: Qualitative spectrographic analysis on material from Daggett County, Utah

$\begin{array}{ll}\text { Major } & \mathrm{Cu} \mathrm{V} \text { Si } \\ \text { Minor } & \mathrm{Ba} \mathrm{AI} \\ \text { Trace } & \mathrm{Ca} \mathrm{Mg} \mathrm{Nb} \mathrm{Fe}\end{array}$

Occurrence and associated minerals: Coating joint and fracture surfaces in sandstone, with gypsum.

Identification: The distinction between volborthite and calciovolborthite is not well established. X-ray powder pattern.

Locality:

Radium No. 5 mine, Slick Rock district. 
Crystal system: Orthorhombic (?)

Habit: As scaly aggregates; also fibrous to dense

Physical properties:

Color: yellow green, olive green Fluorescence: none

Luster: vitreous to pearly on the cleavage

Cleavage: perfect in one direction Hardness: $3 \frac{1}{2}$ Specific gravity:

Strongest lines of X-ray powder pattern: S 7.2, M 2.88, M 2.56, M 2.39, M 1.51

Optical properties:

$\begin{array}{lrll} & \underline{\mathrm{n}} & \text { Pleochroism } & \\ \mathrm{X} & 2.00 & \text { Brown } & \\ \mathrm{Y} & 2.01 & \text { Brown } & \\ \mathrm{Z} & 2.02 & \text { Green } & \text { Biaxial negative } \\ \text { 2V large; } \mathrm{r}>\mathrm{v} \text { strong } & & \end{array}$

Occurrence and associated minerals:

Coating on sandstone, with tyuyamunite and conichalcite.

Identification: The distinction between calciovolborthite and volborthite is not well established.

$\mathrm{X}$-ray powder pattern.

Localities:

Richardson Basin, Moab district. 
Table 2.-Iist of mine names showing county and state

Mine or mine group

County

State

Arrowhead mine

Mesa

Colorado

Bitter Creek mine

Black Mama mine

Blue Jay claim

Buckhorn claim

Montrose

Mesa

San Juan

Colorado

Colorado

San Miguel

Utah

Cactus Rat group

Camp Bird No. 13 mine

Grand

Emery

Cato Sells mine

Cobalt No. 2 mine

Corvusite mine

Crabapple claim

Craven Canyon

Apache

Grand

Colorado

Denise No. 1 mine

Grand

San Juan

Fall River

Utah

Utah

Arizona

Utah

Utah

Utah

South Dekota

Emery

Utah

Frey No. 4 mine

San Juan

Utah

Gray Dawn mine

Gypsum Valley

San Juan

Utah

San Miguel

Colorado

Happy Jack mine

Haystack Mountain area

Hideout (Tiger) mine

Hillside mine

San Juan

Utah

McKinley

San Juan

Yavapai

Huskon No. 2 claim

Coconino

New Mexico

Utah

Arizona

Arizona

Jo Dandy mine

Juniper claim

Montrose

Grand

Colorado

Utah

Iaguas (area)

Valencia

Mesa.

Ia Sal No. 2 mine

Iittle Muriel

Lucky Strike No. 2 mine

Iusk

San Miguel

Emery

Niobrara

Markey No. 3 mine

Marshbank Canyon mine

San Juan

Emery

Matchless mine

Mesa

McCoy group

Grand.

Apache

Apache

Montrose

Apache

New Mexico

Colorado

Colorado

Utah

Wyoming

Utah

Utah

Colorado

Utah

Arizona

Arizona

Mesa No. 5 mine

Mill No. 1 mine

Monument No. 2 mine

Arizona. 
Table 2.--List of mine names showing county and state--Continued

\begin{tabular}{|c|c|c|}
\hline Mine or mine group & County & State \\
\hline $\begin{array}{l}\text { North Point-Gonway claim } \\
\text { North Star mine } \\
\text { Notch mine }\end{array}$ & $\begin{array}{l}\text { San Juan } \\
\text { Montrose } \\
\text { San Juan }\end{array}$ & $\begin{array}{l}\text { Utah } \\
\text { Colorado } \\
\text { Utah }\end{array}$ \\
\hline $\begin{array}{l}\text { Opera Box mine } \\
\text { Oyler mine }\end{array}$ & $\begin{array}{l}\text { Montrose } \\
\text { Wayne }\end{array}$ & $\begin{array}{l}\text { Colorado } \\
\text { Utah }\end{array}$ \\
\hline $\begin{array}{l}\text { Pay Day mine } \\
\text { Placervilie } \\
\text { Polar Mesa } \\
\text { Posey mine } \\
\text { Pumpkin Buttes }\end{array}$ & $\begin{array}{l}\text { Emery } \\
\text { San Miguel } \\
\text { Grand } \\
\text { San Juan } \\
\text { Campbell }\end{array}$ & $\begin{array}{l}\text { Utah } \\
\text { Colorado } \\
\text { Utah } \\
\text { Utah } \\
\text { Wyoming }\end{array}$ \\
\hline $\begin{array}{l}\text { Radium No. } 5 \text { mine } \\
\text { Rex No. } 2 \text { mine } \\
\text { Richardson Basin. }\end{array}$ & $\begin{array}{l}\text { San Miguel } \\
\text { Emery } \\
\text { Grand. }\end{array}$ & $\begin{array}{l}\text { Colorado } \\
\text { Utah } \\
\text { Utah }\end{array}$ \\
\hline $\begin{array}{l}\text { Shinarump No. I mine } \\
\text { Skyline mine } \\
\text { Small Spot mine } \\
\text { Sodarol1 claim } \\
\text { Spring Creek in Brushy Basin }\end{array}$ & $\begin{array}{l}\text { Grand } \\
\text { San Juan } \\
\text { Mesa } \\
\text { San Juan } \\
\text { San Juan }\end{array}$ & $\begin{array}{l}\text { Utah } \\
\text { Utah } \\
\text { Colorado } \\
\text { Utah } \\
\text { Utah }\end{array}$ \\
\hline $\begin{array}{l}\text { Temple Mountain } \\
\text { Thom claim }\end{array}$ & $\begin{array}{l}\text { Emery } \\
\text { Grand. }\end{array}$ & $\begin{array}{l}\text { Utah } \\
\text { Utah }\end{array}$ \\
\hline $\begin{array}{l}\text { White Canyon No. I mine } \\
\text { Whitney mine } \\
\text { Wild Steer mine }\end{array}$ & $\begin{array}{l}\text { San Juan } \\
\text { Montrose } \\
\text { Montrose }\end{array}$ & $\begin{array}{l}\text { Utah } \\
\text { Colorado } \\
\text { Colorado }\end{array}$ \\
\hline Yellow Cat group & Grand & Utah \\
\hline
\end{tabular}


Axelrod, J.Mo, Grimaldi, F。S。, Milton, C., and Murata, Ko J。, 195l, The uranium minerals from the Hillside mine, Yavapai County, Ariz.: Am. Mineralogist, $v .36, p .1-22$.

Barnes, $W_{0} H_{0}$, and Qurashi, M。 Mo, 1952, Unit cell and space group data for certain vanadium minerals: Am. Mineralogist, v. 37, p. 407-422.

Fischer, R. Po, Haff, J。 Co, Rominger, J. F。, 1947, Vanadium deposits near Placerville, San Miguel County, Colo.: Colorado Sci. Soc. Proc., $\mathrm{v}$. 15, p。115-134。

Frondel, J.W., and Fleischer, Michael, 1952, A glossary of uranium- and thorium-bearing minerals: U.S. Geol。 Survey Circular 194.

George, d'Arey, 1949, Mineralogy of uranium and thorium minerals: RMO-563.

Grimaldi, Fo S., May, Irving, and Fletcher, Mo H., 1952, U. S. Geological Survey fluorimetric methods of uranium analysis: U. S。Geol. Survey Circular 199.

Hess, F。I., 1925, New and known minerals from the Utah-Colorado carnotite region: U.S. Geol. Survey Bull. 750, p. 63-78.

Hess, F。 Lo, and Henderson, E. Po, 1931, Fervanite, a hydrous ferric vanadate: Am. Mineralogist, vo $16, \mathrm{p}$. 273-277.

Hess, F。 Lo, and Schaller, Wo To, 1914, Pintadoite and uvanite, two new vanadium minerals from Utah, a preliminary note: Washington Acad. Sci. Jour., v. 4, p. 576-579.

Palache, Charles, Berman, Harry, and Frondel, Clifford, 1944, Dana!s system of mineralogy, 7th ed, , v。 1 and 2, John Wiley and Sons, Inc., New York.

Stern, To Wo, and Weeks, A。 Do, 1952, Second occurrence of bayleyite in the United States: Am. Mineralogist, v. 37, p..1058-1060.

Stich, Jo No, 1953, Spectrographic identification of mineral grains: U. S. Geol. Survey Circular 234.

Weeks, A. D., Cisney, E. A., and Sherwood, A. M., 1950, Humerite and montroseite, two new vanadium minerals from Montrose County, Colo. (abs。): Geol. Soc. America Bull., v。61, p. 1513.

\section{UNPUBLISHED REPORTS}

Shoemaker, E. M., and Luedke, R. G., 1952, Map of the uranium region of the Colorado Plateau: U.S. Geol. Survey Trace Elements Inv. Rept。 279.

Waring, C. L。, and Annell, C. S., 1952, A semiquantitative spectrographic method for the analysis of minerals, rocks, and ores (II): U.S. Geol. Survey Trace Elements Inv. Rept。 215. 